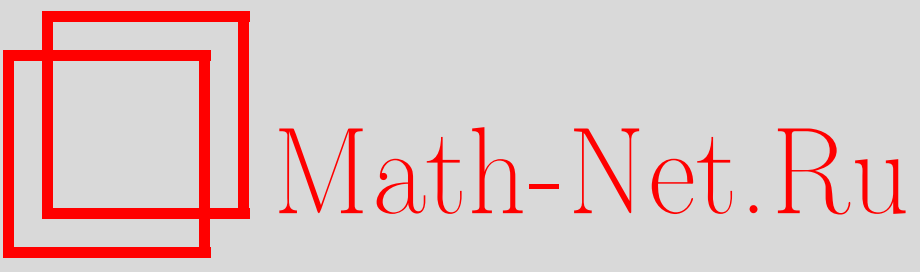

Ю. А. Дубинский, Об аналитических "краевых" задачах на плоскости, УМН, 1997, том 52, выпуск 3, 53-104

DOI: https://doi.org/10.4213/rm849

Использование Общероссийского математического портала Math-Net.Ru подразумевает, что вы прочитали и согласны с пользовательским соглашением

http://www.mathnet.ru/rus/agreement

Параметры загрузки:

IP : 44.207 .124 .84

26 апреля 2023 г., 13:34:23 


\title{
ОБ АНАЛИТИЧЕСКИХ “КРАЕВЫХ” ЗАДАЧАХ НА ПЛОСКОСТИ
}

\author{
Ю. А. Дувинский
}

\section{СОДЕРЖАНИЕ}

Введение . . . . . .

$\S 1$. Аналитическое продолжение решений линейных уравнений $\ldots \ldots \ldots \ldots . \quad 57$

$\S 2$. Принцип конформной эквивалентности многоточечных задач ....... 60

$\S 3$. Аналитическая нелинейная периодическая задача . ............... 65

$\S 4$. Аналитическая нелинейная задача без краевых условий $\ldots . \ldots \ldots \ldots . .76$

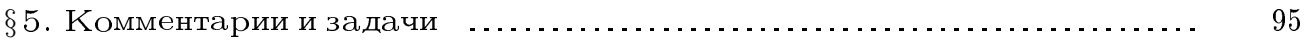

$\S 6$. Об одной задаче продолжения с наименьшим коаналитическим укло-

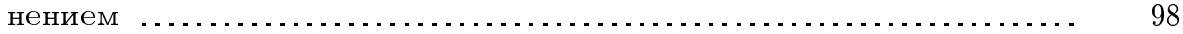

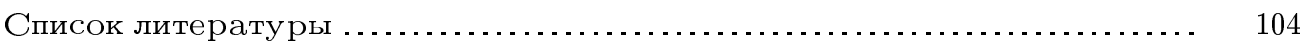

\section{Введение}

В настояшем обзоре изложены результаты последних лет, относяшиеся к теории краевых задач комплексной математической физики или, что то же в нашем тексте, к теории краевых задач для дифференщиальных уравнений с комплексными аргументами.

Строго говоря, как слово “краевые", так и слова “комплексная математическая физика" должны быть взяты в кавычки и не только потому, что к настояшему времени такой теории в четко сформулированном виде не существует, но и потому, что даже самые первые результаты в этом направлении показывают, что естественные краевые задачи для уравнений с комплексными аргументами по самой постановке сушественно отличаются от традиционных краевых задач математической физики и потому требуют собственной терминологии.

Чисто внешне (по крайней мере для автора) необходимость построения теории краевых задач комплексной математической физики обусловлена следуюшим наблюдением.

Как известно, в теории вешественной математической физики среди всевозможных задач принято выделять в отдельную главу задачу Коши как простейшую краевую задачу для эволюционных уравнений по переменной $t \in \mathbb{R}^{1}$, играющей роль времени.

В случае комплексных аргументов выделенная переменная $t \in \mathbb{C}^{1}$ теряет физический смысл времени, и тем самым понятие эволюционности в обычном смысле исчезает. 
Тем не менее, аналитическая теория задачи Коши - теория Коши-Ковалевской получила глубокое и всестороннее развитие. Более того, вместе с экспоненциальной теорией для псевдодифференциальных уравнений с комплексными аргументами (см. [13], [14]) сложился единый комплекс задачи Коши для уравнений с комплексными аргументами.

Совсем иное положение с комплексными краевы.ми задачами, вопрос о постановке которых, насколько нам известно, пока не рассматривался. Совершенно понятно, что прямой перенос постановок основных краевых задач вещественной математической физики на комплексный случай невозможен, поэтому первоочередной задачей в этом направлении является проблема формирования самих математических моделей краевых задач для дифференциальных уравнений с комплексными аргументами (в особенности это касается, как мы увидим ниже, нелинейных аналитических краевых задач).

С этой целью мы предлагаем два подхода для постановок и исследования комплексных краевых задач.

1. Аналитическое продолжение решений краевых задач вешественной математической физики (аналитическое продолжение краевых задач).

2. Минимизация функционалов в пространствах Соболева аналитических функций (вариационньй подход).

Коль скоро реализуем первьй подход, мы получаем, что в пространствах аналитических функций корректны те комплексные краевые задачи, сужение которых на вешественные подпространства являются корректными вешественньми задачами, и наоборот, продолжение вешественных краевых задач дает корректную комплексную задачу. Таким образом, при первом подходе постановка комплексной задачи по форме та же, что и в вешественном случае, и речь идет о ее рассмотрении в пространствах аналитических функций комплексных аргументов.

При втором же подходе, как выяснилось, возникают принципиально новые постановки задач, особенность которых в первую очередь в том, что соответствующее уравнение Эйлера задачи минимизации аналитического функционала содержит в качестве неизвестных не только аналитическую функцию, но и дополнительную неаналитическую функцию - коаналитический потенциал. (Последнее объясняется тем, что в точке минимума градиент аналитического функционала определяется с точностью до произвольного слагаемого, ортогонального подпространству аналитических функций.) Таким образом, второй подход приводит к существенно иньм аналитическим моделям краевых задач математической физики.

Формально оба подхода пригодны для исследования задач математической физики в случае произвольного числа независимых переменных $z_{1}, \ldots, z_{n}$, однако строгое обоснование весьма различно для $n=1$ и $n>1$.

В настояший момент завершенные результаты получены только в одномерном случае, т.е. для уравнений на комплексной плоскости. Эти результаты мы и представляем читателю в настоящей работе. (Что же касается многомерных задач, то с первыми теоремами по нелинейным аналитическим задачам для эллиптических и параболических уравнений можно познакомиться по работам [16], [20].)

Непосредственное содержание обзора следуюшее.

В $\S 1$ и $\S 2$ реализуется первьй подход (аналитическое продолжение краевых вещес- 
твенных задач) применительно к комплексной многоточечной задаче

$$
\begin{gathered}
f^{(n)}(z)+\sum_{k=0}^{n-1} a_{k}(z) f^{(k)}(z)=h(z), \quad z \in G \\
f^{(k)}\left(z_{j}\right)=f_{k j}, \quad j=1, \ldots, m, \quad k=0,1, \ldots, k_{j}-1,
\end{gathered}
$$

где $z_{1}, \ldots, z_{m}(m \leqslant n)$ - произвольные точки в области $G$ или на ее границе, $k_{1}+\cdots+k_{m}=n ; f_{k j}-$ произвольные комплексные числа.

Основной результат - принцип конформной эквивалентности многоточечных задач - гласит, что любая задача (1), (2) “конформно” эквивалентна некоторой вешественной многоточечной задаче на интервале $(a, b)$

$$
\begin{gathered}
g^{(n)}(x)+\sum_{k=0}^{n-1} b_{k}(x) g^{(k)}(x)=q(x), \\
g^{(k)}\left(x_{j}\right)=g_{k j}, \quad j=1, \ldots, m, \quad k=0,1, \ldots, k_{j}-1,
\end{gathered}
$$

где $x_{j} \in(a, b), j=1, \ldots, m$.

Тем самым исследование всякой комплексной задачи (1), (2) адекватно исследованию вешественной (не обязательно аналитической) задачи (3), (4).

Ключевым моментом этого подхода является теорема 1.1 об аналитическом продолжении решений линейных обыкновенных дифференциальных уравнений с интервала $(a, b)$ в комплексную плоскость. В частности, из этой теоремы следует, что если с самого начала точки $z_{1}, z_{2}, \ldots, z_{m}$ лежат на вещественной оси, то все решения комплексной задачи (1), (2) суть аналитические продолжения решений вешественной задачи (3), (4), где

$$
b_{k}(x)=a_{k}(x+i 0), \quad q(x)=h(x+i 0),
$$

т.е. решений сужения исходной комплексной задачи на вещественную ось.

Второму (вариашионному) подходу посвяшены $\S 3$ и $\S 4$, в которых рассмотрены нелинейная аналитическая задача с периодическими условиями и нелинейная аналитическая задача без каких-бы то ни было условий на границе.

Как оказывается, уравнение Эйлера аналитического функционала в пространстве периодических функций имеет вид

(5) $\sum_{k=0}^{m}(-1)^{k} \frac{\partial^{k}}{\partial x^{k}} A_{k}\left(z, f(z), \ldots, f^{(m)}(z)\right)+\partial z r(z)=h(z), \quad z \in\left(y_{-}, y_{+}\right) \times(-\pi, \pi)$,

где $\partial_{z}=(\partial / \partial x-i \partial / \partial y) / 2$ есть оператор Коши-Римана; при этом

$$
f(z+2 \pi)=f(z), \quad r(z+2 \pi)=r(z), \quad r\left(x+i y_{ \pm}\right)=0 .
$$

Здесь наряду с неизвестной аналитической функцией $f(z)$ появляется дополнительная неаналитическая (также неизвестная) функция $r(z)$, играющая роль "невязки" между функциональной и “поточечной” записями равенства нулю вариации функционала. 
При вьводе периодической модели (5), (6) центральную роль играет ортогональное разложение

$$
L_{2}=\mathscr{O}_{2}+\partial_{z} \stackrel{\stackrel{1}{W}}{2}, 1
$$

где $\mathscr{O}_{2}$ - подпространство аналитических функций, суммируемых с квадратом, а $\stackrel{\circ}{W} \underset{2}{1,1}$ - пространство Соболева функций, периодических с периодом $2 \pi$ и равных нулю при $y=y_{ \pm}$.

Именно задача (5), (6) с двумя неизвестными функциями $f(z)$ и $r(z)$ является естественной нелинейной аналитической задачей вариационного типа на классе периодических функщий.

С аналогичных позищий в $\S 4$ исследуется нелинейная аналитическая задача, возникающая при минимизации функционала в пространстве Соболева $\mathscr{O}_{2}^{m}$ аналитических функций в односвязной области $G$ (никаких дополнительных условий на функции не налагаются). В этом случае уравнение Эйлера имеет более сложный вид

$$
\sum_{k=0}^{m}\left(\partial_{\Gamma}^{*}\right)^{k} A_{k}\left(z, f(z), \ldots, f^{(m)}(z)\right)+\partial_{z} r(z)=h(z),
$$

где $r(z)=\stackrel{\circ}{W} \frac{1}{2}(G)$ есть по-прежнему коаналитический потенциал, определяемый ортогональным разложением

$$
L_{2}=\mathscr{O}_{2}+\partial_{z} \stackrel{\circ}{W} \underset{2}{1},
$$

а $\partial_{\Gamma}$ - дифференциальньй оператор первого порядка (конграничньй оператор), определяемьй “геометрий” гранищы $Г$ области $G$.

Отметим, что доказательство ортогональных разложений (7), (8) сводится к решению краевых эллиптических задач для уравнения Коши-Римана

$$
\frac{1}{2}\left(\frac{\partial}{\partial x}-i \frac{\partial}{\partial y}\right) r(z)=q(z)
$$

либо при условиях

$$
r(z+2 \pi)=r(z), \quad r\left(x+i y_{ \pm}\right)=0
$$

(периодический случай), либо при условиях Дирихле

$$
\left.r(z)\right|_{\Gamma}=0
$$

(случай отсутствия краевых условий в исходной задаче минимизации). Обе эти задачи нормально разрешимы по Хаусдорфу, т.е. однозначно разрешимы тогда и только тогда, когда

$$
q(z) \perp \operatorname{Ker} \partial_{\bar{z}}
$$

или, что то же, когда правая часть ортогональна подпространству аналитических функций. (В скобках заметим, что аналогичная теорема для аналитических функций дает окончательньй ответ на вопрос о корректности известной $\bar{\partial}$-проблемы Неймана на плоскости, которьй, насколько нам известно, оставался открытым). 
Завершая введение, отметим, что, познакомившись с содержанием статьи, читатель скорее всего задаст себе больше вопросов, чем найдет ответов, поэтому часть возможных вопросов, решение которых нам представляется достаточно важным, сформулирована в виде задач. Автор будет признателен за любую информацию по затронутым проблемам.

В процессе подготовки статьи для меня весьма полезным были беседы с О. А. Олейник, А.Г. Витушкиным, Е. П. Долженко, М. А. Евграфоовьм, которым я выражаю искреннюю благодарность.

В заключение благодарю Российский фонд фундаментальных исследований (проекты 93-011-1723, 96-01-00621) и Американское математическое обшество за поддержку.

\section{$\S$ 1. Аналитическое продолжение решений линейных уравнений}

\section{1. Постановка задачи.}

Пусть $f_{0}(x):(a, b) \rightarrow \mathbb{C}^{1}$ есть решение линейного дифференциального уравнения порядка $n \geqslant 1$

$$
f_{0}^{(n)}(x)+\sum_{k=0}^{n-1} a_{k}(x) f_{0}^{(k)}(x)=h(x), \quad x \in(a, b),
$$

где коэффициенты $a_{k}(x), k=0,1, \ldots, n-1$, и правая часть $h(x)$ суть непрерывные функции на интервале $(a, b)$, а решение $f_{0}(x) \in \mathbb{C}^{n}(a, b)$.

Пусть, далее, $G$ - односвязная область комплексной плоскости $\mathbb{C}^{1}, z=x+i y$, содержащая интервал $(a, b)$ или примькающая к этому интервалу.

Предположим, что функции $a_{k}(x)$ и $h(x)$ допускают аналитические продолжения в указанную область $G$, т.е. сушествуют аналитические в области $G$ функции $a_{k}(z)$ и $h(z)$ такие, что для всех $x \in(a, b)$

$$
a_{k}(x+i 0) \equiv \lim _{y \rightarrow 0} a_{k}(z)=a_{k}(x), \quad h(x+i 0) \equiv \lim _{y \rightarrow 0} h(z)=h(x)
$$

(в случае, если интервал $(a, b)$ является граничным интервалом для области $G$, то, естественно, либо $y \rightarrow+0$, либо $y \rightarrow-0)$.

Возникает вопрос: допускает ли аналитическое продолжение в область $G$ решение $f_{0}(x)$ уравнения (1.0), т.е. существует ли аналитическая в области $G$ функщия $f(z)$ такая, что

$$
f^{(n)}(z)+\sum_{k=0}^{n-1} a_{k}(z) f^{(k)}(z)=h(z), \quad z \in G,
$$

причем

$$
f(x+i 0)=f_{0}(x), \quad x \in(a, b) .
$$

Прежде чем дать ответ на этот вопрос, отметим, что коль скоро аналитические продолжения $a_{k}(z)$ и $h(z)$ сушествуют, то в соответствии с классическими результатами по проблеме единственности аналитических функций (см., в частности, известную теорему единственности М. и $\Phi$. Риссов [1, с. 1101]) они единственны и, значит, уравнение (1.1) является единственньм аналитическим продолжением исходного 
уравнения (1.0). Следовательно, поставленный вопрос о продолжении решений уравнения (1.0) как решений уравнений (1.1), во-первых, является корректным и, во-вторых, если он имеет положительньй ответ, то по тем же причинам искомое продолжение будет также единственньм. Таким образом, все дело сводится к вопросу существования продолжения $f(z)$.

\section{2. Теоремы продолжения.}

Рассмотрим вначале частный случай области $G$, а именно случай прямоугольника.

ТЕОРема 1.1. Пусть функиии $a_{k}(x), h(x)$, определенные на интервале $(a, b)$, допускают аналитические продолэсения $a_{k}(z), h(z)$ в область $G=\{x \in(a, b)$, $y \in(c, d)\}$, где $c \leqslant 0, d \geqslant 0$ - произвольные числа, причем $c<d$. Тогда существует аналитическая в $G$ функиия $f(z)$, являющаяся решением уравнения (1.1) и такая, что $f(x+i 0)=f_{0}(x), x \in(a, b)$.

ДОкАЗАТЕльСТво. Определим в области $G$ функщию $f(z) \equiv u(x, y)+i v(x, y)$ как решение задачи Коши (по $y \in(c, d)$ )

$$
\begin{gathered}
L(f) \equiv(-i)^{n} f_{y}^{(n)}(z)+\sum_{k=0}^{n-1}(-i)^{k} a_{k}(z) f_{y}^{(k)}(z)=h(z), \\
f_{y}^{(k)}(x+i 0)=i^{k} f_{0}^{(k)}(x), \quad k=0,1, \ldots, n-1,
\end{gathered}
$$

где $x \in(a, b)$ рассматривается как параметр, а $f_{y}(z)$ означает дифференщирование по $y$. Ясно, что если функция $f(z)$ будет аналитична по $z=x+i y$, то она и окажется искомым аналитическим продолжением, ибо в этом случае $f_{y}^{(k)}(z)=i^{k} f^{(k)}(z)$ и уравнение (1.2) совпадает с уравнением (1.1).

Как известно, для доказательства аналитичности $f(z)$ надлежит установить, что $f(z)$ есть дифференцируемая функция в смысле $\mathbb{R}_{x, y}^{2}$ и при этом выполнены условия Коши-Римана.

С этой целью, прежде всего, заметим, что решение задачи (1.2), (1.3) можно, очевидно, представить в виде

$$
f(z)=f_{h}(x, y)+\sum_{k=0}^{n-1} w_{k}(x, y)(i)^{k} f_{0}^{(k)}(x)
$$

где $f_{h}(x, y)$ - решение уравнения (1.2), отвечаюшее нулевым данным Коши при $y=0$, а функции $w_{0}(x, y), \ldots, w_{n-1}(x, y)$ образуют фундаментальную систему решений однородного уравнения, отвечающую “кронекеровским” данным Коши, т.е.

$$
L\left(w_{j}\right)=0, \quad y \in(c, d) ; \quad w_{j}^{(k)}(x, 0)=\delta_{j k}, \quad k, j=0,1, \ldots, n-1 .
$$

В соответствии с классическими результатами об аналитической зависимости решения задачи Коши от параметра функции $f_{h}(x, y), w_{0}(x, y), \ldots, w_{n-1}(x, y)$ аналитичны по $x \in(a, b)$ (разумеется, эти функции аналитичны и по основному аргументу $y$ при $y \neq 0)$. Как известно, функция, аналитическая по двум переменньм порознь, аналитична и по совокупности этих переменных. Поэтому, учитывая формулу (1.4) и включение $f_{0}(x) \in \mathbb{C}^{n}(a, b)$, немедленно получаем, что $f(z) \in \mathbb{C}^{1}(G)$ как функция 
двух переменных $x$ и $y$. Следовательно, $f(z)$ дифференцируема в области $G$ в смысле $\mathbb{R}_{x, y}^{2}$, что нам и требовалось.

Обратимся к условиям Коши-Римана. Для этого положим $w(z)=\partial_{\bar{z}} f(z)$, где, как обычно,

$$
\partial_{\bar{z}}=\frac{1}{2}\left(\frac{\partial}{\partial x}+i \frac{\partial}{\partial y}\right)
$$

и покажем, что $w(z) \equiv 0$ в области $G$.

Действительно, так как $a_{k}(z)$ и $f(z)$ аналитичны в области $G$, т.е. $\partial_{\bar{z}} a_{k}(z) \equiv 0$, то

$$
\partial_{\bar{z}} L(f) \equiv L\left(\partial_{\bar{z}} f\right)
$$

и, следовательно, в силу аналитичности $h(z)$ из уравнения (1.2) получаем, что

$$
L(w)=0, \quad y \in(c, d),
$$

при этом, повторяем, $x \in(a, b)$ - параметр. Далее, учитывая (1.3) и тот факт, что $f(z)$ является решением уравнения (1.0), немедленно находим, что

$$
\left.w(z)\right|_{y=0}=0, \ldots,\left.w^{(n-1)}(z)\right|_{y=0}=0 .
$$

Таким образом, $w(z) \equiv 0$, что вместе с предыдушим и доказьвает аналитичность $f(z)$ в области $G$. Теорема доказана.

В качестве следствий отсюда вытекают следующие результаты.

Теорема 1.2. Всякое решение $f_{0}(x)$ уравнения (1.0), определенное на какомлибо интервале $(a, b) \subset \mathbb{R}^{1}$, аналитически продолжается как решение уравнения (1.1) всюду, куда продолжаются коэффициенты и правая часть этого уравнения.

Для доказательства достаточно заметить, что в предыдуших рассуждениях $x$ и $y$ можно поменять ролями.

Tеорема 1.3. Всякое решение уравнения (1.1), определенное в некоторой подобласти $G_{0} \subset G$, аналитически продолжсается в полную область $G$.

Этот результат известен (см., например, [2]).

ЗАмЕчАниЕ (о единственности). Как уже отмечалось, единственность найденного продолжения $f(z)$ вытекает из известных теорем о единственности аналитических функций. В нашем случае, однако, легко установить единственность, минуя ссылки на эти теоремы, что мы и сделаем с целью замкнутости изложения.

Достаточно, очевидно, доказать, что при $f_{0}(x) \equiv 0$ и $h(x) \equiv 0$ единственным продолжением является функция $f(z) \equiv 0$.

Действительно, пусть $f_{01}(x), \ldots, f_{0 n}(x), x \in(a, b),-$ фундаментальная система решений однородного уравнения

$$
f_{0}^{(n)}(x)+\sum_{k=0}^{n-1} a_{k}(x) f_{0}^{(k)}(x)=0 .
$$


Тогда соответствующая система аналитических продолжений $f_{1}(z), \ldots, f_{n}(z)$, построенных, например, методом, указанным при доказательстве теоремы 1.1, образует фундаментальную систему решений однородного уравнения

$$
f^{(n)}(z)+\sum_{k=0}^{n-1} a_{k}(z) f^{(k)}(z)=0
$$

в области $G$. (В противном случае линейная зависимость этой системы в области $G$ немедленно приводила бы по непрерывности к линейной зависимости системы решений $f_{01}(x), \ldots, f_{0 n}(x)$ на интервале $(a, b)$.) Следовательно, любое наперед заданное продолжение решения $f_{0}(x) \equiv 0$, будучи решением однородного уравнения $(1.5)$, представляется в виде

$$
f(z) \equiv c_{1} f_{1}(z)+\cdots+c_{n} f_{n}(z), \quad z \in G
$$

где $c_{1}, \ldots, c_{n}-$ некоторые постоянные. Отсюда при $y \rightarrow 0$ имеем

$$
0 \equiv c_{1} f_{01}(x)+\cdots+c_{n} f_{0 n}(x), \quad x \in(a, b) .
$$

Тем самьм $c_{1}=0, \ldots, c_{n}=0$ и, значит, $f(z) \equiv 0$. Это и требовалось.

\section{§ 2. Принцип конформной эквивалентности многоточечных задач}

\section{1. Случай “краевых” условий внутри области.}

Пусть $z_{1}, \ldots, z_{m}$ - некоторые точки области $G \subset \mathbb{C}_{z}^{1}$. Рассмотрим задачу о нахождении аналитического в области $G$ решения следуюшей многоточечной задачи:

$$
\begin{aligned}
& f^{(n)}(z)+\sum_{k=0}^{n-1} a_{k}(z) f_{0}^{(k)}(z)=h(z),
\end{aligned}
$$

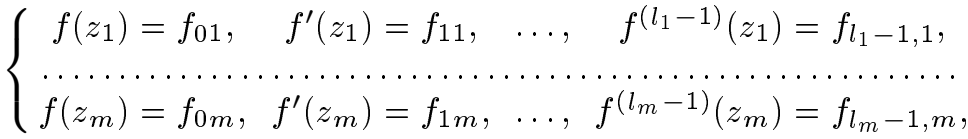

$m \leqslant n, l_{1}+\cdots+l_{m}=n$, где $f_{01}, \ldots, f_{l_{m}-1, m}-$ произвольные комплексные числа.

Основная теорема 1.2 позволяет высказать принцип конформной эквивалентности задач (2.1), (2.2) аналогичным вещественным задачам на интервале $(a, b)$. Вначале будет доказана

Лемма 2.1. Пусть $z_{1}, \ldots, z_{m}-$ произвольный набор точек в области G. Тогда существует аналитическая функция $w=w(z)$, определенная в некоторой односвязной подобласти $G_{0} \subset G$, содержащей эти точки, и такая, что

1) $w^{\prime}(z) \neq 0, z \subset G_{0}$;

2) образ $W_{0}=\operatorname{Im} G_{0}$ при отображении $w=w(z)$ содержит некоторый отрезок $[a, b]$ вещественной оси комплексной плоскости $w=u+i v$;

3) точки $w\left(z_{1}\right)=u_{1}, \ldots, w\left(z_{m}\right)=u_{m}$ принадлежсат интервалу $(a, b)$. 
ДокАЗАТЕЛЬСТво. Через точки $z_{1}, \ldots, z_{m}$ проведем в области $G$ простую аналитическую кривую $\Gamma=\{z=z(u), a \leqslant u \leqslant b\}$ такую, что $z^{\prime}(u) \neq 0, a<u<b$ (сушествование такой кривой будет установлено ниже). Поскольку $z(u)-$ аналитическая функция на отрезке $[a, b]$, то на комплексной плоскости $w=u+i v$ сушествует некоторая окрестность $W_{0}$ этого отрезка, на которую она продолжается как аналитическая функция $z=z(w)$, причем $z^{\prime}(w) \neq 0, w \in W_{0}$. Следовательно, при отображении $z=z(w)$ образ окрестности $W_{0}$ дает некоторую односвязную окрестность $G_{0}$ кривой $\Gamma$. Это и есть искомая подобласть области $G$. Ясно, что обратная функция $w=w(z)$ и определяет требуемое в лемме отображение.

Нам остается установить существование аналитической кривой $\Gamma$, содержащейся в области $G$ и проходящей через точки $z_{1}=x_{1}+i y_{1}, \ldots, z_{m}=x_{m}+i y_{m}$.

Удобно для этого, не ограничивая обшности, считать область $G$ квадратом $\{-1<x<1,-1<y<1\}$, при этом $-1<x_{1}<x_{2}<\cdots<x_{m}<1$.

Очевидно, существует полином $P(x)$, график которого при $|x|<1$ лежит в рассматриваемом квадрате и который принимает приближенно в точках $x_{j}(1 \leqslant j \leqslant m)$ значение $y_{j}$ с любой наперед заданной точностью. Обозначим $\varepsilon_{j}=y_{j}-P\left(x_{j}\right)$ и положим

$$
L(x)=\sum_{j=1}^{m} \varepsilon_{j} l_{j}(x)
$$

где

$$
l_{j}(x)=\prod_{k \neq j} \frac{x-x_{k}}{x_{j}-x_{k}}
$$

(интерполяционньй полином Лагранжа). Ясно, что график многочлена

$$
Q(x)=P(x)+L(x), \quad|x|<1,
$$

приходит через точки $z_{1}, \ldots, z_{m}$ и при малых $\varepsilon_{j}$ остается также внутри квадрата.

Следовательно, функция $z(u) \equiv u+i Q(u),-1<u<1$, и есть искомая функция, определяюшая аналитическую кривую $\Gamma$.

Лемма доказана полностью.

ЗАмечАниЕ 2.1. Отметим, что в частном случае $G=G_{z}^{1}$, когда нет надобности контролировать график $\Gamma$, ответ дает график функции $z(u)=v+i L(u)$, где

$$
L(u)=\sum_{j=1}^{m} y_{j} l_{j}(u)
$$

есть интерполяционный полином Лагранжа.

Вернемся к рассмотрению многоточечной задачи (2.1), (2.2), предполагая, что коэффициенты $a_{k}(z)$ и правая часть $h(z)$ аналитична в области $G$.

В соответствии с доказанной леммой сушествуют односвязная подобласть $G_{0}$, содержашая точки $z_{1}, \ldots, z_{m}$, и невырожденное конформное преобразование, которое 
сводит задачу $(2.1),(2.2)$ в подобласти $G_{0}$ к эквивалентной задаче в некоторой окрестности $W_{0}$ интервала $(a, b)$ :

$$
\begin{aligned}
& g^{(n)}(z)+\sum_{k=0}^{n-1} b_{k}(z) g^{(k)}(z)=k(z), \quad z \in W_{0}, \\
& \left\{\begin{array}{c}
g\left(x_{1}\right)=g_{01}, \quad g^{\prime}\left(x_{1}\right)=g_{11}, \ldots, \quad g^{\left(l_{1}-1\right)}\left(x_{1}\right)=g_{l_{1}-1,1}, \\
\ldots \ldots \ldots \ldots \ldots \ldots \ldots \ldots \ldots \ldots \ldots \ldots \ldots \ldots \ldots \ldots \ldots \ldots \ldots \ldots \ldots \ldots \ldots \ldots \ldots \ldots \ldots \\
g\left(x_{m}\right)=g_{0 m}, \quad g^{\prime}\left(x_{m}\right)=g_{1 m}, \ldots, g^{\left(l_{m}-1\right)}\left(x_{m}\right)=g_{l_{m}-1, m},
\end{array}\right.
\end{aligned}
$$

где $x_{1}, \ldots, x_{m}$ - фиксированные точки на интервале $(a, b)$ (мы не меняем обозначения независимой переменной $z)$.

Более того, в силу теоремы 1.2 аналитическая многоточечная задача (2.3), (2.4) с данньми на интервале $(a, b)$ эквивалентна обычной вешественной многоточечной задаче с аналитическими коэффиициентами $b_{k}(x)$ и правой частью $k(x)$ в пространстве $\mathbb{C}^{n}(a, b)$ :

$$
\begin{aligned}
& g^{(n)}(x)+\sum_{k=0}^{n-1} b_{k}(x) g^{(k)}(x)=k(x), \quad x \in(a, b), \\
& \left\{\begin{array}{c}
g\left(x_{1}\right)=g_{01}, \quad g^{\prime}\left(x_{1}\right)=g_{11}, \ldots, \quad g^{\left(l_{1}-1\right)}\left(x_{1}\right)=g_{l_{1}-1,1}, \\
\ldots \ldots \ldots \ldots \ldots \ldots \ldots \ldots \ldots \ldots \ldots \ldots \ldots \ldots \ldots \ldots \ldots \ldots \ldots \ldots \ldots \ldots \ldots \ldots \ldots \ldots \ldots \\
g\left(x_{m}\right)=g_{0 m}, \quad g^{\prime}\left(x_{m}\right)=g_{1 m}, \ldots, \quad g^{\left(l_{m}-1\right)}\left(x_{m}\right)=g_{l_{m}-1, m} .
\end{array}\right.
\end{aligned}
$$

Именно, любое решение вешественно-аналитической задачи (2.5), (2.6) после аналитического продолжения в некоторую окрестность $W_{0}$ и последующей конформной замены переменной определяет аналитическое в $G_{0}$ решение многоточечной задачи $(2.1),(2.2)$. И наоборот, всякое аналитическое в области $G_{0}$ решение комплексно-аналитической задачи $(2.1),(2.2)$ после некоторого невырожденного конформного преобразования и сужения на вешественную ось дает решение вешественно-аналитической задачи (2.5), (2.6). Ясно, что это соответствие между решениями взаимно однозначно.

Остается заметить, что, поскольку всякое аналитическое в подобласти $G_{0}$ решение уравнения (2.1) продолжается в исходную область $G$ (теорема 1.3 ), отмеченное взаимно однозначное соответствие имеет место и между решениями первоначальной задачи $(2.1),(2.2)$ и решениями вешественной задачи $(2.5),(2.6)$.

В конечном итоге мы приходим к утверждению, которое формулируется как

ПРИНЦИП КОНФОРмНОЙ ЭКВИВАЛЕНТНОСТИ. Совокупность всевозможных комплексно-аналитических многоточечных задач вида (2.1), (2.2) конформно эквивалентна совокупности всех вещественно-аналитических задач вида $(2.5),(2.6)$.

Итак, ввиду принципа конформной эквивалентности изучение аналитических многоточечных задач в комплексной области всегда может быть сведено к изучению вещественно-аналитических задач на интервале. Тем самым известные результаты по вешественно-аналитической теории многоточечных задач переносятся и на комплексно-аналитическую теорию. Это касается, прежде всего, таких вопросов, как корректность поставленных задач, теория спектра, фредгольмовость и др. Какими свойствами по этим вопросам обладает вешественная задача, точно такими же свойствами обладает и комплексная задача. 


\section{2. Случай “краевых" условий на границе области.}

Рассмотрим теперь многоточечную задачу $(2.1),(2.2)$ в случае расположения точек $z_{1}, \ldots, z_{m}$ на границе $Г$ области $G$. Будем считать в этом случае, что функции $a_{k}(z)$ и $h(z)$ аналитичны в области $G$ и непрерывны вплоть до границы $\Gamma$, а решение $f(z)$ непрерьвно вплоть до границы вместе с производными до порядка $n$.

Как известно, если граница Г достаточно гладкая (что и будем предполагать), то существует конформное отображение $w=w(z)$ области $G$ на верхнюю полуплоскость такое, что на $\Gamma$ (за исключением некоторой точки $z_{0}$, образ которой равен бесконечности) сушествуют граничные значения как самой функции $w(z)$, так и всех ее производных $w^{\prime}(z), \ldots, w^{(n)}(z)$. При этом $w^{\prime}(z) \neq 0, z \in \bar{G} \backslash\left\{z_{0}\right\}^{1}$. Тем самым задача $(2.1),(2.2)$ эквивалентна аналогичной многоточечной задаче:

$$
\begin{aligned}
& g^{(n)}(z)+\sum_{k=0}^{n-1} b_{k}(z) g^{(k)}(z)=k(z), \quad \operatorname{Im} z>0, \\
& \left\{\begin{array}{c}
g\left(x_{1}\right)=g_{01}, \quad g^{\prime}\left(x_{1}\right)=g_{11}, \ldots, \quad g^{\left(l_{1}-1\right)}\left(x_{1}\right)=g_{l_{1}-1,1}, \\
\ldots \ldots \ldots \ldots \ldots \ldots \ldots \ldots \ldots \ldots \ldots \ldots \ldots \ldots \ldots \ldots \ldots \ldots \ldots \ldots \ldots \ldots \ldots \ldots \ldots \ldots \ldots \ldots \ldots \\
g\left(x_{m}\right)=g_{0 m}, \quad g^{\prime}\left(x_{m}\right)=g_{1 m}, \ldots, \quad g^{\left(l_{m}-1\right)}\left(x_{m}\right)=g_{l_{m}-1, m}
\end{array}\right.
\end{aligned}
$$

где $x_{1}, x_{2}, \ldots, x_{m}$ - образы $z_{1}, z_{2}, \ldots, z_{m}$. При этом коэффициенты $b_{k}(z)$ и $h(z)-$ аналитические функции в верхней полуплоскости и непрерьвные вплоть до границы $y=0$ (как и ранее, мы не изменяем обозначения независимой переменной).

В соответствии с теоремой 1.1 эта задача эквивалентна вещественной задаче:

$$
\begin{aligned}
& g^{(n)}(x)+\sum_{k=0}^{n-1} b_{k}(x) g^{(k)}(x)=k(x), \quad x \in(a, b), \\
& \left\{\begin{array}{c}
g\left(x_{1}\right)=g_{01}, \quad g^{\prime}\left(x_{1}\right)=g_{11}, \quad \ldots, \quad g^{\left(l_{1}-1\right)}\left(x_{1}\right)=g_{l_{1}-1,1}, \\
\ldots \ldots \ldots \ldots \ldots \ldots \ldots \ldots \ldots \ldots \ldots \ldots \ldots \ldots \ldots \ldots \ldots \ldots \ldots \ldots \ldots \ldots \ldots \ldots \ldots \ldots \ldots \ldots \ldots \ldots \\
g\left(x_{m}\right)=g_{0 m}, \quad g^{\prime}\left(x_{m}\right)=g_{1 m}, \ldots, \quad g^{\left(l_{m}-1\right)}\left(x_{m}\right)=g_{l_{m}-1, m},
\end{array}\right.
\end{aligned}
$$

где $(a, b)$ - произвольньй интервал, содержаший $x_{1}, \ldots, x_{m}$.

Таким образом, принцип конформной эквивалентности комплексных и вешественных многоточечных задач имеет место и в случае разложения точек $z_{1}, \ldots, z_{m}$ на границе области.

Остается заметить, что и в общем случае расположения точек $z_{1}, \ldots, z_{m}$ как внутри, так и на гранище области принцип конформной эквивалентности также справедлив (действительно, всегда можно взять подобласть области $G$ так, чтобы все точки $z_{1}, \ldots, z_{m}$ оказались на границе этой подобласти, применить принцип конформной эквивалентности, а затем вновь использовать теоремы продолжения).

\footnotetext{
${ }^{1}$ Точные условия таковы. Пусть $z=z(s)(0 \leqslant s \leqslant S)$ - параметрическое задание границы как длины дуги $s$ и $\theta(s)=\arg z^{\prime}(s)$ есть угол между касательным вектором к границе и осью $x$. Тогда, если $\theta(s)$ дифференцируема $(n-1)$ раз и производная $\theta^{(n-1)}(s)$ удовлетворяет условию Гёльдера с показателем $\alpha \in(0,1]$, то при подходе к границе $\Gamma$ существуют предельные граничные значения функций $w(z), \ldots, w^{(n)}(z)$. При этом $n$-я производная также удовлетворяет на $\Gamma$ условию Гёльдера с показателем $\alpha$.
} 


\section{3. Случай "краевых" условий на прямой.}

Особенно просто вьглядит принцип конформной эквивалентности многоточечных задач при расположении точек $z_{1}, \ldots, z_{m}$ на одной прямой, которую без ущерба общности можно считать вешественной осью. В этом случае комплексная многоточечная задача является просто аналитическим продолжением вещественной задачи, и наоборот, сужение комплексной задачи есть вешественная задача.

ПРИмеР 2.1. Рассмотрим двуточечную задачу

$$
\begin{gathered}
-\frac{d}{d z}\left(a(z) \frac{d f}{d z}\right)+q(z) f=h(z), \quad z \in G, \\
f(a)=0, \quad f(b)=0 .
\end{gathered}
$$

Известно, что соответствующая вешественная задача

$$
\begin{gathered}
-\frac{d}{d x}\left(a(x) \frac{d f}{d x}\right)+q(x) f=h(x), \quad x \in(a, b), \\
f(a)=0, \quad f(b)=0,
\end{gathered}
$$

фредгольмова, спектр ее дискретен. В частности, при $\operatorname{Re} a(x) \geqslant a_{0}>0, \operatorname{Re} q(x) \geqslant$ $q_{0}>0$ задача $(2.9),(2.10)$ однозначно разрешима при любой правой части.

Следовательно, всеми этими свойствами обладает и комплексная задача $(2.7),(2.8)$, причем соответствуюшие решения получаются из решений задачи $(2.9),(2.10)$ аналитическим продолжением.

ПримеР 2.2. Рассмотрим комплексную задачу Валле-Пуссена

$$
\begin{gathered}
f^{(n)}(z)+\sum_{k=0}^{n-1} a_{k}(z) f^{(k)}(z)=h(z), \quad z \in G, \\
f\left(x_{1}\right)=f_{1}, \ldots, f\left(x_{n}\right)=f_{n},
\end{gathered}
$$

где $x_{1}<x_{2}<\cdots<x_{n}$ - фиксированные точки на некотором интервале $(a, b)$.

Как известно (см., например, [12], [1, с. 573]), вешественная задача Валле-Пуссена

$$
\begin{gathered}
f^{(n)}(x)+\sum_{k=0}^{n-1} a_{k}(x) f^{(k)}(x)=h(x), \quad x \in(a, b), \\
f\left(x_{1}\right)=f_{1}, \ldots, f\left(x_{n}\right)=f_{n},
\end{gathered}
$$

корректна, если вьполнено условие

$$
\sum_{k=0}^{n-1} \sup \left|a_{n-k}(x)\right| \frac{(b-a)^{k}}{k !}<1
$$

Следовательно, условие (2.11) достаточно и для корректности комплексной задачи Валле-Пуссена. 


\section{§. Аналитическая нелинейная периодическая задача}

3.1. Необходимые пространства. Основное ортогональное разложение.

3.1.1. Определения. Пусть $f(z)$ - комплексная функция, определенная в полосе $y^{-}<\operatorname{Im} z<y^{+}\left(y^{-}, y^{+}-\right.$произвольные числа), периодическая с периодом $2 \pi$. Пусть, далее, $G=\left\{x \in(-\pi, \pi), y \in\left(y^{-}, y^{+}\right)\right\}$- основной прямоугольник периодичности.

Обозначим через $L_{2} \equiv L_{2}(G)$ гильбертово пространство указанных функций, суммируемых с квадратом, т.е.

$$
L_{2}=\left\{f(z): f(z+2 \pi) \equiv f(z) \&\|f(z)\| \equiv \iint_{G}|f(z)|^{2} d x d y<\infty\right\}
$$

скалярное произведение в $L_{2}$ вводится стандартно, т.е. $\langle f, g\rangle_{L_{2}} \equiv \iint_{G} f(z) \bar{g}(z) d x d y$.

Далее обозначим через $\mathscr{O}_{2}$ подпространство пространства $L_{2}$, состоящее из аналитических периодических функций $f(z)$, суммируемых с квадратом в области $G$, т.е.

$$
\mathscr{O}_{2}=\left\{f(z): f(z) \in L_{2} \& \partial_{\bar{z}} f(z) \equiv 0\right\},
$$

где, как обычно, $\partial_{\bar{z}}=\frac{1}{2}(\partial / \partial x+i \partial / \partial y)$.

Наша ближайшая цель - описать пространство $\mathscr{O}_{2}$ и его ортогональное дополнение $\mathscr{O}_{2}^{\perp}$, что необходимо в дальнейшем для постановки нелинейной периодической задачи в пространствах аналитических функщий.

3.1.2. Базисность системы экспонент. Прежде всего, отметим, что при любых $y^{-}$и $y^{+}$функции $\exp i n z(n=0, \pm 1, \ldots)$ образуют ортогональную систему в $\mathscr{O}_{2}$. Более того, справедлива

Tеорема 3.1 (ср. [3, с. 324]). Система функиий $e^{i n z}, n=0, \pm 1, \ldots$, образует в $\mathscr{O}_{2}$ базис Фурье, т.е. любая функиия $f(z) \in \mathscr{O}_{2}$ представляется рядом

$$
f(z)=\sum_{n=-\infty}^{\infty} C_{n} e^{i n z}
$$

При этом коэффициенты $C_{n}$ могут быть вычислены по одной из формул:

$$
\begin{gathered}
C_{n}=\frac{n}{\pi\left(e^{-2 n y^{-}}-e^{-2 n y^{+}}\right)} \iint_{G} f(z) e^{-i n \bar{z}} d x d y \quad(n \neq 0), \\
C_{0}=\frac{1}{2 \pi\left(y^{+}-y^{-}\right)} \iint_{G} f(z) d x d y,
\end{gathered}
$$

илu

$$
C_{n}=\frac{1}{2 \pi} \int_{-\pi}^{\pi} f(x+i 0) e^{-i n x} d x
$$

(для удобства считаем, что $\left.y^{-}<0<y^{+}\right)$. 
ДокАЗАТЕльство. Действительно, так как при любом фиксированном $y \in$ $\left(y^{-}, y^{+}\right)$, очевидно, $f(x+i y) \in L_{2}(-\pi, \pi)$, то

$$
f(x+i y)=\sum_{n=-\infty}^{\infty} C_{n}(y) e^{i n x},
$$

где

$$
C_{n}(y)=\frac{1}{2 \pi} \int_{-\pi}^{\pi} f(x+i y) e^{-i n x} d x .
$$

Используя интегральную теорему Коши и условие периодичности, имеем

$$
\begin{aligned}
C_{n}(y) & =\frac{1}{2 \pi} \int_{-\pi}^{\pi} f(x+i y) e^{-i n(x+i y)} d x e^{-n y} \\
& =\frac{1}{2 \pi} \int_{-\pi}^{\pi} f(x+i 0) e^{-i n x} d x e^{-n y}=C_{n}(0) e^{-n y} \equiv C_{n} e^{-n y} .
\end{aligned}
$$

Таким образом, ряд (3.4) принимает вид искомого ряда Фурье (3.1) в $\mathscr{O}_{2}$, при этом коэффициенты Фурье вычисляются по формулам (3.3). Ясно (равенство Парсеваля и теорема Лебега о предельном переходе под знаком интеграла), что ряд (3.4) сходится к $f(z)$ в смысле $L_{2}(G)$.

Остается заметить, что в силу теоремы Коши

$$
\begin{aligned}
\left\langle f(z), e^{i n z}\right\rangle_{G} & \equiv \iint_{G} f(x+i y) e^{-i n \bar{z}} d x d y \\
& =\int_{y^{-}}^{y^{+}}\left(\int_{-\pi}^{\pi} f(x+i y) e^{-i n(x+i y)} d x\right) e^{-2 n y} d y \\
& =2 \pi C_{n}(0) \int_{y^{-}}^{y^{+}} e^{-2 n y} d y=C_{n} \frac{\pi}{n}\left(e^{-2 n y^{-}}-e^{-2 n y^{+}}\right),
\end{aligned}
$$

что и дает формулы (3.2). Теорема доказана.

В рамках обсуждения доказанной теоремы сделаем несколько замечаний.

Во-первых, отметим, что если область $G$ симметрична относительно начала координат, т.е. $y^{+}=-y^{-}=y_{0}>0$, то, как и в случае вешественных рядов Фурье, нечетные функции разлагаются в ряд по системе $\sin n z, n=0,1,2, \ldots$, а четные - по системе $\cos n z, n=0,1,2, \ldots$, при этом

$$
C_{n}=\frac{n}{\pi \operatorname{sh} 2 n y_{0}}\left\langle f(z), \begin{array}{c}
\sin n z \\
\cos n z
\end{array}\right\rangle_{G} .
$$

Во-вторых, если область $G$ является полуполосой, т.е. либо $y^{-}=-\infty$, либо $y^{+}=+\infty$, то соответствующие ряды Фурье определяются только частью экспонент. Именно, в первом случае базис образует система $\exp i n z$ при $n>0$, а во втором случае - при $n<0$.

Наконец, отметим, что случай полосы, т.е. случай, когда одновременно $y^{-}=-\infty$, а $y^{+}=+\infty$, бессодержателен, ибо в этом случае, как можно показать, пространство $\mathscr{O}_{2}$ тривиально, т.е. состоит из одного нуля. 
3.1.3. Формула $\mathscr{O}_{2}^{\perp}=\partial_{z} \stackrel{\circ}{W}^{1,1}$. Обратимся к подпространству $\mathscr{O}_{2}$. Для этой цели будем использовать пространства Соболева функций $r(z): G \rightarrow \mathbb{C}^{1}$, рассматриваемых как комплекснозначные функции вещественных переменных $x \in \mathbb{R}^{1}$ и $y \in\left(y^{-}, y^{+}\right)$, периодические по $x$ с периодом $2 \pi$. Именно, положим

$$
W_{2}^{1,1}=\left\{r(z):\|r(z)\|_{1} \equiv\|r(z)\|_{0}+\left\|r_{x}^{\prime}(z)\right\|_{0}+\left\|r_{y}^{\prime}(z)\right\|_{0}<\infty, r(z+2 \pi)=r(z)\right\},
$$

где, напоминаем, $\|\cdot\|_{0}-$ норма в $L_{2}$.

Наконец, примем

$$
\stackrel{\circ}{W}_{2}^{1,1}=\left\{r(z): r(z) \in W_{2}^{1,1}, r\left(x+i y^{+}\right)=0, r\left(x+i y^{-}\right)=0\right\} .
$$

Теорема 3.2. Имеет место формула

$$
\mathscr{O}_{2}^{\perp}=\partial_{z} \stackrel{\circ}{W}_{2}^{1,1}
$$

где $\partial_{z}=\frac{1}{2}(\partial / \partial x-i \partial / \partial y)$. Более точно, $q(z) \in \mathscr{O}_{2}^{\perp}$ тогда и только тогда, когда существует (единственная) функиия $r(z) \in \stackrel{\circ}{W_{2}^{1,1}}$ такая, что

$$
q(z)=\partial_{z} r(z), \quad z \in G .
$$

ДОКАЗАТЕЛЬСТВО. Пусть $q(z) \in \mathscr{O}_{2}^{\perp}$, т.е. $q(z) \in L_{2}$ и при этом

$$
\left\langle q(z), e^{i n z}\right\rangle_{G}=0, \quad n=0, \pm 1, \ldots
$$

Нетрудно проверить, что если $q(z)$ представить в виде ряда Фурье

$$
q(z)=\sum_{n=-\infty}^{\infty} q_{n}(y) e^{i n x}
$$

то условия (3.5) эквивалентны условиям

$$
\int_{y^{-}}^{y^{+}} q_{n}(y) e^{-n y} d y=0, \quad n=0, \pm 1, \ldots
$$

Для нахождения функции $r(z)$, указанной в теореме, рассмотрим краевую задачу (вообще говоря, переопределенную):

$$
\begin{gathered}
\partial_{z} r(z)=q(z), \\
r(x+2 \pi+i y)=r(x+i y), \quad x \in \mathbb{R}^{1}, \quad y \in\left(y^{-}, y^{+}\right), \\
r\left(x+i y^{-}\right)=0, \quad r\left(x+i y^{+}\right)=0
\end{gathered}
$$

и будем искать ее решение в виде

$$
r(z)=\sum_{n=-\infty}^{\infty} r_{n}(y) e^{i n x} .
$$


Ясно, что проекции $r_{n}(y)$ являются решениями краевых задач

$$
\begin{aligned}
r_{n}^{\prime}(y)-n r_{n}(y) & =2 i q_{n}(y), \quad y \in\left(y^{-}, y^{+}\right), \\
r_{n}\left(y^{-}\right) & =0, \quad r_{n}\left(y^{+}\right)=0 .
\end{aligned}
$$

Решение задачи $\left(3.7^{n}\right),\left(3.9^{n}\right)$ задается формулой

$$
r_{n}(y)=2 i \int_{y^{-}}^{y} q_{n}(\eta) e^{-n \eta} d \eta e^{n y}
$$

(отметим, что $r_{n}\left(y^{+}\right)=0$ в силу условия (3.6)). Остается получить оценку, показывающую, что $r(z) \in W_{2}^{1,1}(G)$.

Имеем $(n \neq 0)$ :

$$
\begin{aligned}
\left\|r_{n}(y)\right\|_{L_{2}\left(y^{-}, y^{+}\right)}^{2} & =4 \int_{y^{-}}^{y^{+}}\left|\int_{y^{-}}^{y} q_{n}(\eta) e^{-n \eta} d \eta\right|^{2} e^{2 n y} d y \\
& =\frac{2}{n} \int_{y^{-}}^{y^{+}}\left|\int_{y^{-}}^{y} q_{n}(\eta) e^{-n \eta} d \eta\right|^{2} \frac{d}{d y} e^{2 n y} d y \\
& \leqslant \frac{4}{n} \int_{y^{-}}^{y^{+}}\left|\int_{y^{-}}^{y^{+}} q_{n}(\eta) e^{-n \eta} d \eta\right| e^{n y}\left|q_{n}(y)\right| d y \\
& \equiv \frac{2}{n} \int_{y^{-}}^{y^{+}}\left|r_{n}(y)\right| \cdot\left|q_{n}(y)\right| d y \\
& \leqslant \frac{2}{n}\left\|r_{n}(y)\right\|_{L_{2}\left(y^{-}, y^{+}\right)} \cdot\left\|q_{n}(y)\right\|_{L_{2}\left(y^{-}, y^{+}\right)},
\end{aligned}
$$

откуда немедленно заключаем, что

$$
\left\|r_{n}(y)\right\|_{L_{2}\left(y^{-}, y^{+}\right)} \leqslant \frac{2}{n}\left\|q_{n}(y)\right\|_{L_{2}\left(y^{-}, y^{+}\right)} .
$$

Используя теперь равенство Парсеваля и уравнение $\left(3.7^{n}\right)$, получаем, что $r(z) \in$ $W_{2}^{1,1}$, причем

$$
\|r(z)\|_{W_{2}^{1,1}(G)} \leqslant M\|q(z)\|_{L_{2}(G)}
$$

где $M>0-$ постоянная.

Итак, представление функции $q(z) \in \mathscr{O}_{2}^{\perp}$ в виде $q(z)=\partial_{z} r(z)$ получено.

Обратно, пусть функция $q(z) \in L_{2}$ имеет вид $q(z)=\partial_{z} r(z)$, где $r(z) \in \stackrel{\circ}{W_{2}^{1,1}}$. Тогда, интегрируя по частям, находим

$$
\langle q(z), \exp i n z\rangle_{G}=\left\langle\partial_{z} r(z), \exp i n z\right\rangle_{G}=-\left\langle r(z), \partial_{\bar{z}} \exp i n z\right\rangle_{G}=0,
$$

т.е. $q(z) \in \mathscr{O}_{2}^{\perp}$. Теорема доказана. 
ЗАмЕчАниЕ 3.1. Теорема 3.2 справедлива и для случая полосы $G=\{-\pi<x<\pi$, $\left.y^{-}<y<+\infty\right\}$, при этом в условиях (3.9) сохраняется только условие $r\left(z+i y^{-}\right)=0$. Доказательство проводится по той же схеме, но дополнительно надо заметить, что $r_{n}(y) \rightarrow 0$ при $y \rightarrow+\infty$. Именно, в силу условий $(3.6)$ (при $\left.y^{+} \rightarrow+\infty\right)$

$$
\begin{aligned}
\left|r_{n}(y)\right| \equiv\left|2 e^{n y} \int_{y}^{\infty} q_{n}(\eta) e^{-n y} d \eta\right| & \leqslant 2 e^{n y}\left(\int_{y}^{\infty}\left|q_{n}(\eta)\right|^{2} d \eta\right)^{1 / 2}\left(\int_{y}^{\infty} e^{-2 n \eta} d \eta\right)^{1 / 2} \\
& =\sqrt{\frac{2}{n}}\left(\int_{y}^{\infty}\left|q_{n}(\eta)\right|^{2} d \eta\right)^{1 / 2} \rightarrow 0
\end{aligned}
$$

при $y \rightarrow+\infty$, ибо $q_{n}(y) \in L_{2}\left(y^{-},+\infty\right)$.

Аналогично рассматривается и случай $\left(-\infty, y_{+}\right)$.

Вывод. Итак, всякая функция $f(z) \in L_{2}$ представляется в виде ортогональной суммы аналитической и, скажем, коаналитической составляющих. Именно, $f(z)=$ $f_{a}(z)+f_{c a}$, где $f_{a}(z) \in \mathscr{O}_{2}$, т.е. $\partial_{\bar{z}} f_{a}(z) \equiv 0$, а коаналитическая составляюшая $f_{c a}(z)$ имеет вид $f_{c a}(z)=\partial_{z} r(z)$, где $r(z) \in \stackrel{\circ}{W}_{2}^{1,1}$ (функцию $r(z)$ естественно назвать коаналитическим потенциалом). Коль скоро известна аналитическая часть $f_{a}(z)$ (и тем самым коаналитическая часть $\left.f_{c a}(z)\right)$, то потенщиал $r(z)$ определяется теоремой 3.2 . Что же касается аналитической части $f_{a}(z)$, то в соответствии с теоремой 3.1

$$
f_{a}(z)=\sum_{n=-\infty}^{\infty} C_{n} \exp i n z
$$

где

$$
C_{n}=\frac{n}{\pi\left(e^{-2 n y_{-}}-e^{-2 n y_{+}}\right)}\langle f(z), \exp i n z\rangle_{G} .
$$

Все сказанное и составляет содержание разложения

$$
L_{2}=\mathscr{O}_{2}+\partial_{z} \stackrel{\circ}{W} \stackrel{1,1}{ }
$$

В заключение пункта укажем интегральное представление решения задачи (3.7)-(3.9). Используя (3.6), находим

$$
\begin{aligned}
r(z) & =2 i \sum_{n=-\infty}^{\infty}\left(\int_{y^{-}}^{y} q_{n}(\eta) e^{-n \eta} d \eta\right) e^{n y} e^{i n x} \\
& =2 i \sum_{n=-\infty}^{0} \int_{y^{-}}^{y} q_{n}(\eta) e^{n(y-\eta)} d \eta e^{i n x}-2 i \sum_{n=1}^{\infty} \int_{y^{-}}^{y^{+}} q_{n}(\eta) e^{n(y-\eta)} d \eta e^{i n x}
\end{aligned}
$$


Такое представление дает возможность суммирования под знаком интеграла. Именно,

$$
\begin{aligned}
r(z)= & \frac{i}{\pi} \sum_{n=-\infty}^{0} \int_{-\pi}^{\pi} \int_{y^{-}}^{y} q(\xi+i \eta) e^{n(y-\eta)+i n(x-\xi)} d \xi d \eta \\
& -\frac{i}{\pi} \sum_{n=1}^{+\infty} \int_{-\pi}^{\pi} \int_{y}^{y^{+}} q(\xi+i \eta) e^{n(y-\eta)+i n(x-\xi)} d \xi d \eta \\
= & \frac{i}{\pi} \int_{-\pi}^{\pi} \int_{y^{-}}^{y} \frac{e^{y-\eta+i(x-\xi)}}{e^{y-\eta+i(x-\xi)}-1} q(\xi+i \eta) d \xi d y \\
& +\frac{i}{\pi} \int_{-\pi}^{\pi} \int_{y}^{y^{+}} \frac{e^{y-\eta+i(x-\xi)}}{e^{y-\eta+i(x-\xi)}-1} q(\xi+i \eta) d \xi d y \\
= & \frac{i}{\pi} \iint_{G} \frac{q(\xi+i \eta) e^{y-\eta+i(x-\xi)}}{e^{y-\eta+i(x-\xi)}-1} d \xi d \eta .
\end{aligned}
$$

Таким образом, решение задачи

$$
\partial_{z} r(z)=q(z), \quad r(z+2 \pi)=r(z), \quad r\left(x+i y^{+}\right)=r\left(x+i y^{-}\right)=0
$$

имеет вид

$$
r(z)=q(z) * \frac{1}{\pi i} \frac{e^{i \bar{z}}}{e^{i \bar{z}}-1}
$$

Поскольку $q(z)$ ортогональна единице, решение $r(z)$ можно записать также в виде

$$
r(z)=q(z) * \frac{1}{\pi i} \frac{1}{e^{i \bar{z}}-1}
$$

3.1.4. Шкала соболевских пространств функций и функционалов. Пусть $\mathscr{O}_{2}^{m}(m \geqslant 1$ - целое) есть пространство Соболева аналитических функций, т.е.

$$
\mathscr{O}_{2}^{m}=\left\{f(z): f(z) \in \mathscr{O}_{2}, \ldots, f^{(m)}(z) \in \mathscr{O}_{2}\right\}
$$

со скалярным произведением

$$
(f, g)_{m}=\sum\left\langle f^{(k)}(z), g^{(k)}(z)\right\rangle_{G}
$$

Ясно, что система $\exp i n z, n=0, \pm 1, \ldots$, образует в $\mathscr{O}_{2}^{m}$ ортогональньй базис.

Далее, пусть $\mathscr{O}_{2}^{-m}=\left(\mathscr{O}_{2}^{m}\right)^{*}-$ сопряженное пространство. Очевидно,

$$
\mathscr{O}_{2}^{m} \subset \cdots \subset \mathscr{O}_{2}^{1} \subset \mathscr{O}_{2} \subset \mathscr{O}_{2}^{-1} \subset \cdots \subset \mathscr{O}_{2}^{-m} \subset \cdots,
$$

причем, как нетрудно видеть, оператор

$$
\Lambda_{2 m} \equiv \sum_{k=0}^{m}(-1)^{k} \frac{d^{2 k}}{d z^{2 k}}
$$


является дуальным оператором пары $\left(\mathscr{O}_{2}^{+m}, \mathscr{O}_{2}^{-m}\right)$, т.е. отображение

$$
\Lambda_{2 m}: \mathscr{O}_{2}^{m} \rightarrow \mathscr{O}_{2}^{-m}
$$

есть изоморфизм, удовлетворяюший равенству

$$
\left\langle\Lambda_{2 m} f, f\right\rangle=(f, f)_{m}=\|f\|_{m}^{2},
$$

где $f(z) \in \mathscr{O}_{2}^{m}$ - произвольная функция.

Отсюда следует, что нормы в $\mathscr{O}_{2}^{-m}$ определяются скалярным произведением

$$
(f, g)_{-m} \equiv\left(\Lambda_{2 m}^{-1} f, \Lambda_{2 m}^{-1} g\right)_{m},
$$

а система экспонент и в пространствах $\mathscr{O}_{2}^{-m}$ также образует ортогональньй базис.

Таким образом, вся шкала пространств (3.10) обладает единым базисом Фурье $\exp i n z, n=0, \pm 1, \ldots$. Отсюда, в частности, следует, что все вложения в цепочке (3.10) являются плотньми.

Далее, с цепочкой пространств аналитических функций (3.10) свяжем “объемлющую" ее цепочку анизотропных соболевских пространств

$$
W_{2}^{+m, 0} \subset \cdots \subset W_{2}^{+1,0} \subset L_{2} \subset W_{2}^{-1,0} \subset \cdots \subset W_{2}^{-m, 0} \subset \cdots,
$$

где

$$
W_{2}^{m, 0}=\left\{q(z): q(z) \in L_{2}, \frac{\partial q}{\partial x}(z) \in L_{2}, \ldots, \frac{\partial^{m} q}{\partial x^{m}} \in L_{2}\right\} .
$$

Очевидно, $\mathscr{O}_{2}^{m} \subset W_{2}^{m, 0}$, причем $\mathscr{O}_{2}^{m}$ является замкнутым подпространством в пространстве $W_{2}^{m, 0}$, более того,

$$
W_{2}^{m, 0}=\mathscr{O}_{2}^{m}+\partial_{z}\left(\nabla^{-1} W_{2}^{m, 0}\right)
$$

где

$$
\nabla^{-1} W_{2}^{m, 0}=\left\{r(z): \nabla r(z) \in W_{2}^{m, 0}, r\left(x+i y^{ \pm}\right)=0\right\} .
$$

Формула (3.12) означает, что всякая функция $f(z) \in W_{2}^{m, 0}$ может быть представлена в виде

$$
f(z)=f_{a}(z)+\partial_{z} r(z)
$$

где $f_{a}(z) \in \mathscr{O}_{2}^{m}$ есть аналитическая часть, а $r(z) \in \nabla^{-1} W_{2}^{m, 0}$ - коаналитический потенциал.

Указанное представление есть следствие теоремы 3.2 и очевидной возможности дифференцировать по $x$ уравнение $\partial_{z} r(z)=q(z)$, оставаясь в рамках периодических функций.

Далее, дуальньм оператором пары $W^{m, 0}, W^{-m, 0}$ является оператор

$$
\widetilde{\Lambda}_{2 m} \equiv \sum_{k=0}^{m}(-1)^{k} \frac{\partial^{2 k}}{\partial x^{2 k}}
$$


являюшийся, очевидно, расширением оператора $\Lambda_{2 m}$. Отсюда следует, что всякий функционал $h(z) \in W_{2}^{-m, 0}$ представляется в виде $h(z)=\widetilde{\Lambda}_{2 m} f(z)$, где $f(z) \in W_{2}^{m, 0}$ - некоторая функция. Следовательно, в соответствии с формулой (3.13)

$$
h(z)=\widetilde{\Lambda}_{2 m} f_{a}(z)+\widetilde{\Lambda}_{2 m}\left(\partial_{z} r(z)\right)=\Lambda_{2 m} f_{a}(z)+\partial_{z} \widetilde{\Lambda}_{2 m} r(z) \equiv h_{a}(z)+h_{c a}(z),
$$

где аналитическая часть $h_{a}(z)$ есть сужение $h(z)$ на $\mathscr{O}_{2}^{m}$, равное нулю на ортогональном дополнении, а коаналитическая часть $h_{c a}(z)$, напротив, есть сужение $h(z)$ на ортогональное дополнение, равное нулю на $\mathscr{O}_{2}^{m}$. При этом коаналитическая часть функционала может быть записана в виде $h_{c a}(z)=\partial_{z} \tilde{r}(z)$, где

$$
\tilde{r}(z) \in \nabla^{-1} W^{-m, 0}=\left\{\tilde{r}(z): \nabla \tilde{r}(z) \in W_{2}^{-m, 0}, \tilde{r}\left(x \pm i y^{ \pm}\right)=0\right\} .
$$

В заключение отметим, что сказанное в этом пункте можно эквивалентно сформулировать в виде теоремы, обобщающей основную теорему 3.2 .

ТЕОрема 3.3. Для любой функции (функционала) $q(z) \in W_{2}^{s, 0}, s=0, \pm 1, \ldots$, удовлетворяющей условиям ортогональности

$$
\langle q(z), \exp i n z\rangle=0, \quad n=0, \pm 1, \ldots,
$$

задача

$$
\partial_{z} r(z)=q(z), \quad r(z+2 \pi)=r(z), \quad r\left(x \pm i y^{ \pm}\right)=0
$$

имеет единственное решение $r(z) \in \nabla^{-1} W_{2}^{s, 0}$.

Тем самым для любых $s=0, \pm 1, \ldots$

$$
W_{2}^{s, 0}=\mathscr{O}_{2}^{s}+\partial_{z}\left(\nabla^{-1} W_{2}^{s, 0}\right) .
$$

3.2. Уравнение Эйлера квадратичного функционала. Нелинейная периодическая задача.

3.2.1. Основная "наводящая" модель. Для того чтобы естественно прийти к аналитической постановке нелинейной периодической задачи, рассмотрим вопрос о минимуме аналитического функционала. Именно, в пространстве $\mathscr{O}_{2}^{1}$ будем минимизировать функционал

$$
J(f) \equiv \operatorname{Re} \iint_{G}\left[\frac{1}{2} a\left(z,\left|f^{\prime}(z)\right|^{2}\right)+\frac{1}{2} b\left(z,|f(z)|^{2}\right)-h(z) \bar{f}(z)\right] d x d y
$$

где $h(z) \in L_{2}-$ некоторая функция, а периодические по $z$ функции $a(z, s), b(z, s)$ $(z \in G, s \geqslant 0)$ удовлетворяют неравенствам

$$
\begin{gathered}
a_{0} s-k_{0} \leqslant \operatorname{Re} a(z, s) \leqslant A_{0} s+K_{0}, \\
b_{0} s-m_{0} \leqslant \operatorname{Re} b(z, s) \leqslant B_{0} s+M_{0},
\end{gathered}
$$

$\left(0<a_{0}<A_{0}<\infty, 0<b_{0}<B_{0}<\infty, k_{0}, K_{0}, m_{0}, M_{0}\right.$ - некоторые постоянные) и имеют ограниченные производные по $s$. (Очевидно, при этих условиях функционал $J(f)$ 
определен на всем пространстве $\mathscr{O}_{2}^{1}$ и полуограничен снизу.) Как известно, в точке минимума вариация функционала

$\delta J(f) \varphi(z) \equiv \operatorname{Re} \iint_{G}\left[a_{s}^{\prime}\left(z,\left|f^{\prime}(z)\right|^{2}\right) f^{\prime}(z) \overline{\varphi^{\prime}(z)}+b_{s}^{\prime}\left(z,|f(z)|^{2}\right) f(z) \overline{\varphi(z)}-h(z) \varphi(z)\right] d x d y=0$ для любой функции $\varphi(z) \in \mathscr{O}_{2}^{1}$.

Допустим, что $\mathscr{D}_{x} a^{\prime}\left(z,\left|f^{\prime}(z)\right|^{2}\right) f^{\prime}(z) \in L_{2}$. Тогда, учитывая аналитичность $\varphi(z)$ и интегрируя по частям в первом слагаемом, получаем тождество

$$
\operatorname{Re} \iint_{G}\left[-\mathscr{D}_{x}\left(a^{\prime}\left(z,\left|f^{\prime}(z)\right|^{2}\right) f^{\prime}(z)\right)+b^{\prime}\left(z,\left|f^{\prime}(z)\right|^{2}\right) f(z)-h(z)\right] \bar{\varphi}(z) d x d y=0,
$$

которое справедливо не только для $\varphi(z) \in \mathscr{O}_{2}^{1}$, но и для любой функции $\varphi(z) \in \mathscr{O}_{2}$, ибо, как было ранее отмечено, вложение $\mathscr{O}_{2}^{1} \subset \mathscr{O}_{2}$ всюду плотно.

Следовательно, в соответствии с основной теоремой предыдущего параграфа (теорема 3.2$)$ существует функция $r(z) \in \stackrel{\circ}{W}_{2}^{1,1}$ такая, что

$$
-\mathscr{D}_{x}\left(a^{\prime}\left(z,\left|f^{\prime}(z)\right|^{2}\right) f^{\prime}(z)\right)+b^{\prime}\left(z,|f(z)|^{2}\right) f(z)+\partial_{z} r(z)=h(z),
$$

где равенство понимается в смысле $L_{2}$.

Это и есть уравнение Эйлера для функционала $J(f)$.

Из сказанного видно, что уравнение Эйлера, понимаемое в классическом смысле (равно, впрочем, как и в смысле теории обобщенных функций вещественных переменных), содержит в качестве неизвестных величин две функции $f(z)$ и $r(z)$, первая из которых дает решение исходной задачи минимизации в $\mathscr{O}_{2}^{1}$, а вторая является потенциалом коаналитической “невязки” в уравнении Эйлера $(3.14)^{2}$. Ясно, что если $a_{s}^{\prime}\left(z,\left|f^{\prime}(z)\right|^{2}\right) f^{\prime}(z)$ и $b_{s}^{\prime}\left(z,|f(z)|^{2}\right) f(z)$ суть только измеримые ограниченные функции, то уравнение (3.14) для $f(z) \in \mathscr{O}_{2}^{1}$ и $r(z) \in \nabla^{-1} W_{2}^{-1,0}=\stackrel{\circ}{W}_{2}^{0,1}$ понимается в смысле функционалов над $\stackrel{\circ}{W} \underset{2}{1,0}$.

Итак, мы можем сказать, что необходимое условие экстремума

$$
\delta J(f) \varphi(z)=0, \quad \varphi(z) \in \mathscr{O}_{2}^{1},
$$

эквивалентно либо дифференциальному уравнению

$$
L(f) \equiv-\mathscr{D}_{x}\left[a_{s}^{\prime}\left(z,\left|f^{\prime}(z)\right|^{2}\right) f^{\prime}(z)\right]+b_{s}^{\prime}\left(z,|f(z)|^{2}\right) f(z)=h(z),
$$

понимаемому в смысле аналитических функционалов над $\mathscr{O}_{2}^{1}$, либо дифференциальному уравнению

$$
L(f)+\partial_{z} r(z)=h(z)
$$

понимаемому в смысле обобщенных функций над $\stackrel{\circ}{W_{2}^{1,0}}$. Особо отметим линейньй случай $a(z, s) \equiv a(z) s, b(z, s) \equiv b(z) s$, где $a(z)$ и $b(z)$ - ограниченные аналитические функции в $G$. В этом случае $r(z) \equiv 0$ и уравнение Эйлера принимает вид

$$
-\left(a(z) f^{\prime}(z)\right)^{\prime}+b(z) f(z)=h(z) .
$$

\footnotetext{
${ }^{2}$ Читатель, несомненно, заметил идейную параллель с системой уравнений Навье-Стокса, содержащей потенциальную невязку $\operatorname{grad} p(x)$.
} 
Его, очевидно, можно решать не только в классе аналитических функционалов $\mathscr{O}_{2}^{-1}$, но и в основном пространстве аналитических функций $\mathscr{O}_{2}^{1}$.

3.2.2. Разрешимость нелинейной периодической задачи. Обобщая модельный пример предыдушего пункта, рассмотрим следуюшую задачу: найти решение уравнения

$$
\sum_{k=0}^{m}(-1)^{k} \mathscr{D}_{x}^{k} A_{k}\left(z, f, \ldots, f^{(m)}\right)+\partial_{z} r(z)=h(z), \quad z \in G
$$

при условиях

$$
\begin{gathered}
f(z+2 \pi)=f(z), \\
r\left(x+i y^{-}\right)=0, \quad r\left(x+i y^{+}\right)=0 .
\end{gathered}
$$

Неизвестньми функциями здесь являются аналитическая функция $f(z)$ и коаналитический потенщиал $r(z)$.

Допустим, что вьполнены следуюшие условия:

I. Функции $A_{k}\left(z, \zeta_{0}, \ldots, \zeta_{m}\right), k=0,1, \ldots, m$, непрерывны по $z \in G$ и $\zeta_{0} \in \mathbb{C}^{1}, \ldots$, $\zeta_{m} \in \mathbb{C}^{1}$ и удовлетворяют неравенствам

$$
\left|A_{k}\left(z, \zeta_{0}, \ldots, \zeta_{m}\right)\right| \leqslant M\left(\sum_{l=0}^{m}\left|\zeta_{l}\right|+1\right),
$$

где $M>0$ - постоянная.

II. Условие коэрцитивности. Для любых $z \in G, \zeta_{0} \in \mathbb{C}^{1}, \ldots, \zeta_{m} \in \mathbb{C}^{1}$

$$
\operatorname{Re} \sum_{k=0}^{m} A_{k}\left(z, \zeta_{0}, \ldots, \zeta_{m}\right) \bar{\zeta}_{m} \geqslant a_{0}\left(\left|\zeta_{0}\right|^{2}+\left|\zeta_{m}\right|^{2}\right)-K
$$

где $a_{0}>0, K>0$-постоянные.

ТЕОРема 3.4. Пусть выполнены условия I, II. Тогда для любой правой части $h(z) \in W_{2}^{-m, 0}$ существует по крайней мере одна пара функций $f(z) \in \mathscr{O}_{2}^{m}, r(z) \in$ $\nabla^{-1} W_{2}^{-m, 0}$, удовлетворяющая уравнению (3.15) над пространством $W_{2}^{m, 0}$.

ДокаЗАТЕльство. Сначала найдем функцию $f(z) \in \mathscr{O}_{2}^{m}$, удовлетворяющую уравнению (3.15) над пространством $\mathscr{O}_{2}^{m}$, т.е. такую функцию $f(z) \in \mathscr{O}_{2}^{m}$, что для любой основной функции $\varphi(z) \in \mathscr{O}_{2}^{m}$ справедливо тождество

$$
\sum_{k=0}^{m}\left\langle A_{k}\left(z, f(z), \ldots, f^{(m)}(z)\right), \varphi^{(k)}(z)\right\rangle_{G}=\langle h(z), \varphi(z)\rangle_{G} .
$$

Решение этого интегрального тождества стандартно (метод компактности). Именно, приближенное решение $f_{N}(z), N=1,2 \ldots$, ишем в виде

$$
f_{N}(z)=\sum_{|n| \leqslant N} C_{n} \exp i n z,
$$


при этом постоянные $C_{n}$ определяются из системы нелинейных алгебраических уравнений

$$
\begin{gathered}
\sum_{k=0}^{m}\left\langle A_{k}\left(z, f_{N}(z), \ldots, f_{N}^{(m)}(z)\right),(i n)^{k} \exp i n z\right\rangle_{G}=\langle h, \exp i n z\rangle_{G} \\
n=0, \pm 1, \ldots, \pm N
\end{gathered}
$$

Разрешимость системы $\left(3.18^{n}\right)$ обеспечивается условием коэрцитивности II и известной леммой об остром угле (см., например, [4]-[6]). Таким образом, приближенные решения $f_{N}(z)$ найдены.

Далее, в силу того же условия коэрцитивности для приближенных решений справедлива оценка

$$
\left\|f_{N}(z)\right\|_{m} \leqslant M\left(\|h\|_{-m}+1\right), \quad M>0,
$$

из которой вытекает, что сушествуют функция $f(z) \in \mathscr{O}_{2}^{m}$ и некоторая подпоследовательность приближенных решений (чтобы не менять обозначений, будем по-прежнему писать $\left.f_{N}(z)\right)$ такие, что $f_{N}(z) \rightarrow f(z)$ слабо в $\mathscr{O}_{2}^{m}$.

Поскольку функции $f_{N}(z)$ аналитичны в $G$, можно считать, что $f_{N}(z) \rightarrow f(z)$ равномерно на любом компакте, содержашемся в области $G$. Следовательно, переходя к пределу в $\left(3.18^{n}\right)$ при $N \rightarrow \infty$ (и любом фиксированном $n$ ) и учитывая, что система $\exp i n z, n=0, \pm 1, \ldots$, образует базис в $\mathscr{O}_{2}^{m}$, получаем, что найденная функция $f(z)$ удовлетворяет тождеству (3.18), т.е. является искомым решением.

Остается заметить, что в соответствии с теоремой 3.3 определяется теперь и функция $r(z)$ как решение задачи

$$
\partial_{z} r(z)=q(z), \quad r(z+2 \pi)=r(z), \quad r\left(x+i y^{ \pm}\right)=0,
$$

где $q(z)$ есть коаналитическая составляющая разности

$$
h(z)-\sum_{k=0}^{m} \mathscr{D}_{x}^{k} A_{k}\left(z, f, \ldots, f^{(m)}\right) .
$$

Теорема доказана.

ЗАмЕчАнИЕ (о единственности). Если функции $A_{k}\left(z, \zeta_{0}, \ldots, \zeta_{m}\right)$ таковы, что оператор

$$
A(f) \equiv \sum_{k=0}^{m}(-1)^{k} \mathscr{D}_{x}^{k} A_{k}\left(z, f, \ldots, f^{(m)}\right)
$$

является строго монотонньм оператором из $\mathscr{O}_{2}^{m}$ в $\mathscr{O}_{2}^{-m}$, то решение $(f(z), r(z))$ единственно.

ПримеР 3.1. Пусть $a(s)$ и $b(s)(s \geqslant 0)$ - непрерывные вещественные функции, удовлетворяюшие неравенствам

$$
0<a_{0} \leqslant a(s) \leqslant A_{0}<\infty, \quad 0<b_{0} \leqslant b(s) \leqslant B_{0}<\infty .
$$

Соответствуюшее уравнение

$$
-\mathscr{D}_{x}\left(a\left(\left|f^{\prime}(z)\right|^{2}\right) f^{\prime}(z)\right)+b\left(|f(z)|^{2}\right) f(z)+\partial_{z} r(z)=h(z)
$$

удовлетворяет условию II и, следовательно, данная периодическая задача разрешима. 
ПримеР 3.2. Периодическая задача для уравнения

$$
(-1)^{m} \mathscr{D}_{x}^{m}\left(\left(1+|z|^{2}\right) f^{(m)}(z)\right)+\partial_{z} r(z)=h(z)
$$

имеет единственное решение $f(z) \in \mathscr{O}_{2}^{m}, r(z) \in \nabla^{-1} W_{2}^{-m, 0}$.

Особо отметим линейный случай, в котором не возникает коаналитической невязки. Именно, рассмотрим задачу определения $2 \pi$-периодической аналитической функции $f(z)$, удовлетворяюшей уравнению

$$
\sum_{k=0}^{m}(-1)^{k} \mathscr{D}_{z}^{k}\left(a_{k}(z) \mathscr{D}_{z}^{k} f(z)\right)=h(z),
$$

где $a_{k}(z)$ - аналитические ограниченные функции в области $G$, периодические с периодом $2 \pi$ (ясно, что в этом случае $\mathscr{D}_{x}=\mathscr{D}_{z}$ ).

Теорема 3.5. Если для любы $\zeta_{k} \in \mathbb{C}^{1}, k=0, \ldots, m$,

$$
\operatorname{Re} \sum_{k=0}^{m} a_{k}(z) \zeta_{k} \overline{\zeta_{k}} \geqslant a_{0}\left(\left|\zeta_{0}\right|^{2}+\left|\zeta_{m}\right|^{2}\right)-K
$$

где $a_{0}>0, K>0$ - постоянные, то для любой $h(z) \in \mathscr{O}(G)$ и периодической $c$ периодом $2 \pi$ существует единственное $2 \pi$-периодическое решение $f(z) \in \mathscr{O}(G)$ уравнения (3.19).

ДокАЗАТЕльство. Действительно, всякая функция $h(z) \in \mathscr{O}(G)$ в любой подобласти $G_{\varepsilon}=\left\{-\pi<x<\pi, y^{-}+\varepsilon<y<y^{+}-\varepsilon\right\}$ является, очевидно, ограниченной и, тем более, суммируемой с квадратом. Тем самым по теореме 3.4 в области $G_{\varepsilon}$ существует единственное аналитическое $2 \pi$-периодическое решение $f_{\varepsilon}(z) \in \mathscr{O}_{2}^{m}\left(G_{\varepsilon}\right)$ уравнения (3.19). Ясно, что при $\varepsilon \rightarrow 0$ последовательность $f_{\varepsilon}(z)$ определяет единственную функцию $f(z) \in \mathscr{O}(G)$, которая и есть искомое решение. Это и требовалось.

ПримеР 3.3. Задача

$$
\begin{gathered}
-\frac{d}{d z}\left(a(z) \frac{d f(z)}{d z}\right)+b(z) f(z)=h(z), \\
f(z+2 \pi)=f(z), \quad z \in \mathbb{C}^{1}
\end{gathered}
$$

имеет единственное решение, если $a(z)$ и $b(z)$ суть $2 \pi$-периодические аналитические функции, причем $\operatorname{Re} a(z) \geqslant a_{0}>0, \operatorname{Re} b(z) \geqslant b_{0}>0, z \in G$.

\section{§4. Аналитическая нелинейная задача без краевых условий}

\section{1. Ортогональные разложения.}

$$
\begin{gathered}
L_{2}=\mathscr{O}_{2}+\partial_{z} \stackrel{\circ}{W} \underset{2}{1}, \quad L_{2}=\overline{\mathscr{O}}_{2} \dot{+} \partial_{\bar{z}} \stackrel{\circ}{W}_{2}^{1}, \\
L_{2}=\mathscr{O}_{2} \dot{+} \overline{\mathscr{O}}_{2}+\partial_{z} \partial_{\bar{z}} \stackrel{\circ}{W}_{2}^{2} .
\end{gathered}
$$


4.1.1. Основная формула. Пусть $G \subset \mathbb{C}_{z}^{1}$ есть ограниченная область с границей Г. Обозначим через $L_{2} \equiv L_{2}(G)$ пространство Лебега функций $f(z): G \rightarrow \mathbb{C}^{1}$, суммируемых с квадратом, т.е.

$$
L_{2}=\left\{f(z):\|f(z)\|_{0}^{2} \equiv \iint_{G}|f(z)|^{2} d x d y<\infty\right\}
$$

Далее, пусть

$$
\mathscr{O}_{2}=\left\{f(z): f(z) \in L_{2} \& \partial_{\bar{z}} f(z) \equiv 0\right\}, \quad \partial_{\bar{z}}=\frac{1}{2}\left(\frac{\partial}{\partial x}+i \frac{\partial}{\partial y}\right)
$$

есть пространство функций, аналитических в области $G$ и суммируемых с квадратом в этой области. Очевидно, $\mathscr{O}_{2} \subset L_{2}$ и является замкнутым подпространством.

Прежде чем описать ортогональное дополнение $\mathscr{O}_{2}^{\perp}$, сформулируем основное условие на область $G$. Для этого рассмотрим линейное подмножество $B \subset \mathscr{O}_{2}$ функций, аналитических в замыкании области $G$, т.е.

$$
B=\left\{f(z): \partial_{\bar{z}} f(z) \equiv 0, z \in \bar{G}\right\}
$$

ОПРЕДЕЛЕНИЕ 4.1. Назовем область $G$ допустимой, если вложение $B \subset \mathscr{O}_{2}$ плотно.

Например, допустимой областью является круг $K_{R}=\{z:|z|<R<\infty\}$. Действительно, как нетрудно установить, в этом случае при любом $R>0$ система функций $z^{n}, n=0,1, \ldots$, образует в $\mathscr{O}_{2}$ базис Фурье, т.е. любая функция $f(z) \in \mathscr{O}_{2}$ разлагается в ряд

$$
f(z)=\sum_{n=0}^{\infty} f_{n} z^{n}
$$

где

$$
f_{n}=\frac{n+1}{\pi R^{n+1}} \iint_{K_{R}} f(z) \overline{z^{n}} d x d y \text {. }
$$

Тем самьм, очевидно, вложение $B \subset \mathscr{O}_{2}$ всюду плотно.

Ясно, что допустимой будет и любая область $G$, конформно (причем невырожденно) эквивалентная кругу $K_{R}$. Таковой будет, например, любая односвязная ограниченная область, граница которой удовлетворяет классическому условию Ляпунова. Это немедленно вытекает из известного уточнения Келлога основной теоремы теории конформных отображений (см., например, [7, гл. II, §1, п. 29]).

Допустимой областью будет также любое кольцо

$$
K_{r, R}=\left\{z \in \mathbb{C}^{1}: r<|z|<R\right\}
$$

(базис Фурье образует система $\left\{z^{n}\right\}, n=0, \pm 1, \ldots$ ), а также области, конформно эквивалентные кольцу.

Ниже, если не оговорено противное, область $G$ считается допустимой. 
Теорема 4.1. Справедлива формула

$$
\mathscr{O}_{2}^{\perp}=\partial_{z} \stackrel{\circ}{W}_{2}^{1}, \quad \partial_{z} \equiv \frac{1}{2}\left(\frac{\partial}{\partial x}-i \frac{\partial}{\partial y}\right),
$$

где $\stackrel{\circ}{W} \underset{2}{1} \equiv \stackrel{\circ}{W} \frac{1}{2}(G)$ - пространство Соболева функций, равных нулю на Г. Это означает, что $q(z) \in \mathscr{O}_{2}^{\perp}$ тогда и только тогда, когда найдется (единственная) функция $r(z) \in W_{2}^{1}$ такая, что

$$
\partial_{z} r(z)=q(z),\left.\quad r(z)\right|_{\Gamma}=0 .
$$

При этом справедлива оченка

$$
\|r(z)\|_{0}+\left\|r_{x}(z)\right\|_{0}+\left\|r_{y}(z)\right\|_{0} \leqslant M\|q(z)\|_{0}
$$

где $M>0-$ постоянная.

ДокАЗАТЕльство. Достаточность представления (4.1) очевидна. Действительно, пусть $\varphi(z) \in B$. Тогда

$$
\begin{aligned}
\langle q(z), \varphi(z)\rangle_{G} & \equiv \iint_{G} q(z) \overline{\varphi(z)} d x d y=\iint_{G} \partial_{z} r(z) \overline{\varphi(z)} d x d y \\
& =-\iint_{G} r(z) \overline{\partial_{\bar{z}} \varphi(z)} d x d y=0
\end{aligned}
$$

(граничные значения при интегрировании по частям, очевидно, не возникают, ибо $\left.r(z)\right|_{\Gamma}=0$, а $\varphi(z)$ аналитична в $\left.\bar{G}\right)$. Поскольку область $G$ допустима, т.е. вложение $B \subset \mathscr{O}_{2}$ плотно, то $\langle q(z), \varphi(z)\rangle_{G}=0$ для любой функции $\varphi(z) \in \mathscr{O}_{2}$. Это и значит, что $q(z) \in \mathscr{O}_{2}^{\perp}$.

Доказательство необходимости представления (4.1) проведем вначале для частного случая области $G$, а именно для круга $K_{R}$.

Пусть $q(z) \in \mathscr{O}_{2}^{\perp}$, что эквивалентно тому, что

$$
\left\langle q(z), z^{n}\right\rangle_{K_{R}}=0, \quad n=0,1, \ldots
$$

Очевидно, искомая функция $r(z) \in \stackrel{\circ}{W} \underset{2}{1}\left(K_{R}\right)$ должна быть решением эллиптической краевой задачи (переопределенной с точки зрения краевых условий)

$$
\begin{gathered}
\frac{1}{2}\left(\frac{\partial}{\partial x}-i \frac{\partial}{\partial y}\right) r(z)=q(z), \quad|z|<R, \\
\left.r(z)\right|_{|z|=R}=0 .
\end{gathered}
$$

Это решение в полярных координатах $\rho, \theta$ стандартно определяется методом Фурье. Именно, если

$$
q(z)=\sum_{n=-\infty}^{\infty} q_{n}(\rho) e^{i n \theta}
$$


то формально

$$
r(z)=\sum_{n=-\infty}^{\infty} r_{n}(\rho) e^{i n \theta}
$$

где

$$
r_{n}(\rho)= \begin{cases}2 \int_{0}^{\rho} \eta^{n} q_{n-1}(\eta) d \eta \rho^{-n}, & n>0 \\ -2 \int_{\rho}^{R} \eta^{n} q_{n-1}(\eta) d \eta \rho^{-n}, & n \leqslant 0 .\end{cases}
$$

Нетрудно проверить, что условия ортогональности (4.2) эквивалентны равенствам

$$
\int_{0}^{R} \rho^{n} q_{n-1}(\rho) d \rho=0, \quad n=1,2, \ldots
$$

откуда немедленно следует, что $r_{n}(R)=0$ для всех $n=0, \pm 1, \ldots$.

Таким образом, соответствуюший ряд (4.6) является формальным решением задачи $(4.3),(4.4)$.

Остается установить, что для любой функции $q(z) \in \mathscr{O}_{2}^{\perp}$ ряд (4.6) действительно определяет решение $r(z) \in \stackrel{\circ}{W} \underset{2}{1}$ этой задачи. С этой целью заметим, что если $r(z) \in \stackrel{\circ}{W} \underset{2}{1}$ есть решение задачи $(4.3),(4.4)$, то оно является и решением задачи Дирихле

$$
\Delta r(z)=4 \partial_{\bar{z}} q(z),\left.\quad r(z)\right|_{|z|=R}=0 .
$$

Поэтому если $q(z) \in L_{2}$, то для $r(z) \in \stackrel{\circ}{W} \underset{2}{1}$ справедлива известная $L_{2}$-оценка

$$
\|r(z)\|_{0}+\|\nabla r(z)\|_{0} \leqslant M\|q(z)\|_{0}
$$

где $M>0$-постоянная. В частности, если правые части в (4.3) являются частичными суммами ряда (4.5), то соответствуюшие неформальные решения - частичные суммы ряда (4.6) - образуют последовательность, сходящуюся в $\stackrel{\circ}{W} \underset{2}{1}$. Таким образом, для любой функции $q(z) \in \mathscr{O}_{2}^{\perp}$ ряд (4.6) сходится в норме пространства $\stackrel{\circ}{W} \underset{2}{1}$ и тем самым определяет соответствуюшее решение.

Итак, случай $G=K_{R}$ изучен.

ЗАмЕчАНИЕ 4.1. Суммируя ряд (4.6), нетрудно получить интегральное представление решения, а именно

$$
r(z)=\frac{1}{\pi} \iint_{K_{R}} \frac{q(\zeta)}{\bar{z}-\bar{\zeta}} d \xi d \eta, \quad \zeta=\xi+i \eta
$$

Обратимся теперь к случаю произвольной ограниченной области $G$ и покажем, что решение задачи

$$
\begin{gathered}
\partial_{z} r(z)=q(z), \quad q(z) \in \mathscr{O}_{2}^{\perp}, \quad z \in G \\
\left.r(z)\right|_{\Gamma}=0
\end{gathered}
$$


определяется формулой

$$
r(z)=\frac{1}{\pi} \iint_{G} \frac{q(\zeta)}{\bar{z}-\bar{\zeta}} d \xi d \eta, \quad z \in G
$$

Действительно, выберем $R>0$ так, чтобы $G \subset K_{R}$, и положим

$$
q^{*}(z)= \begin{cases}q(z), & z \in G, \\ 0, & z \in K_{R} \backslash G .\end{cases}
$$

Поскольку $q(z)$ ортогональна любой функции $\varphi(z) \in \mathscr{O}_{2}$ в области $G, q^{*}(z)$ ортогональна любой функции $\varphi(z)$ из $\mathscr{O}_{2}$ в круге $K_{R}$. Следовательно, по доказанному соответствуюшее решение $r^{*}(z)$ определяется формулой

$$
r^{*}(z)=\frac{1}{\pi} \iint_{K_{R}} \frac{q^{*}(\zeta)}{\bar{z}-\bar{\zeta}} d \xi d \eta, \quad z \in K_{R}
$$

или, что то же,

$$
r^{*}(z)=\frac{1}{\pi} \iint_{G} \frac{q^{*}(\zeta)}{\bar{z}-\bar{\zeta}} d \xi d \eta, \quad z \in K_{R}
$$

При этом

$$
\|r(z)\|_{W_{2}^{1}\left(K_{R}\right)} \leqslant M\left\|q^{*}(z)\right\|_{L_{2}\left(K_{R}\right)}=M\|q(z)\|_{L_{2}(G)}
$$

где $M>0-$ некоторая постоянная.

Далее, в силу исходных условий ортогональности на функцию $q(z)$ правая часть формулы (4.11) при всех $z \in K_{R} \backslash G$ равна нулю, т.е. $r^{*}(z)$ есть продолжение нулем в круг $K_{R}$ функции $r(z)$, определяемой формулой (4.10). Тем самым $r(z) \in \stackrel{\circ}{W} \underset{2}{1}(G)$ и является решением исходной задачи (4.8), (4.9). Очевидно, оценка (4.12) преврашается в оценку $\|r(z)\|_{W_{2}^{\circ}(G)} \leqslant M\|q(z)\|_{L_{2}(G)}$. Теорема полностью доказана.

ЗАмечАниЕ 4.2. Аналогичное рассуждение показьвает, что если $q(z) \in \mathscr{O}_{2}^{\perp} \cap$ $\stackrel{\circ}{W}_{2}^{m}(G)$, то решение $r(z) \in \stackrel{\circ}{W}_{2}^{m+1}(G)$. Это означает, что в задаче $(4.8),(4.9)$ носители правой части и решений согласованы в том смысле, что продолжение нулем правой части $q(z)$ в более широкую область соответствует нулевому продолжению решения в ту же область.

ЗАмЕчАНИЕ 4.3. Отметим, что классическая разрешимость задачи (4.1), т.е. разрешимость в пространстве $\mathbb{C}^{1}(G) \cap \mathbb{C}(\bar{G})$, анонсирована в работе А. Джураева [8].

Вывод. Подводя итог сказанному, получаем, что произвольная функция $f(z) \in$ $L_{2} \equiv L_{2}(G)$ представляется в виде

$$
f(z)=f_{a}(z)+f_{c a}(z)
$$

где $f_{a}(z) \in \mathscr{O}_{2}$, а $f_{c a}(z) \in \mathscr{O}_{2}^{\perp}$. Естественно назвать функцию $f_{a}(z)$ аналитической частью $f(z)$, а $f_{c a}(z)$ - ее коаналитической частью. При этом коаналитическая 
часть $f_{c a}(z)$ однозначно представима в виде $f_{c a}(z)=\partial_{z} r(z)$, где $r(z) \in \stackrel{\circ}{W} \frac{1}{2}(G)$ - ее (скажем) коаналитический потенциал. Все это мы и обозначаем формулой

$$
L_{2}=\mathscr{O}_{2}+\partial_{z} \stackrel{\circ}{W} \underset{2}{1}
$$

Для дальнейшего отметим, что это представление лежит в основе построения математической модели задачи минимизации функционалов типа нормы в пространствах аналитических функций. Поэтому формулу (4.13) будем называть основнылм разложением.

4.1.2. Разложение $L_{2}=\mathscr{O}_{2}+\overline{\mathscr{O}}_{2}+\partial_{z} \stackrel{\circ}{W}_{2}^{1}$. Установленное в предыдущем пункте основное разложение (4.13) можно развить. Именно, обозначим через $\overline{\mathscr{O}}_{2}$ подпространство антианалитических функций, т.е. функций $f(z) \in L_{2}$, аналитических по $\bar{z}$. Очевидно, что отображение $f(z) \rightarrow \overline{f(z)}$ определяет изоморфизм $\mathscr{O}_{2}$ и $\overline{\mathscr{O}}_{2}$. Ясно также, что, повторяя предыдушие рассуждения, будем иметь разложение

$$
L_{2}=\overline{\mathscr{O}}_{2}+\partial_{\bar{z}} \stackrel{\circ}{W}_{2}^{1},
$$

где $\partial_{\bar{z}}=\frac{1}{2}(\partial / \partial x+i \partial / \partial y)$. Иными словами, всякая функция $q(z) \in L_{2}$, удовлетворяюшая условиям $\left\langle q(z), \bar{z}^{n}\right\rangle=0, n=0,1, \ldots$, однозначно представляется в виде $q(z)=\partial_{\bar{z}} r(z)$, где $r(z) \in \stackrel{\circ}{W} \stackrel{1}{2}$, при этом

$$
\|r(z)\|_{0}+\|\nabla r(z)\|_{0} \leqslant M\|q(z)\|_{0}
$$

где $M>0$ - постоянная.

Далее, считая для $f(z) \in \mathscr{O}_{2}$, что $f(0)=0$, и тем самым устраняя нетривиальное пересечение $\mathscr{O}_{2}$ и $\overline{\mathscr{O}}_{2}$ (очевидно, $\mathscr{O}_{2} \cap \overline{\mathscr{O}}_{2}=\mathbb{C}^{1}$ ), мы можем ставить вопрос об описании ортогонального дополнения к сумме $\mathscr{O}_{2} \dot{+} \overline{\mathscr{O}}_{2}$, т.е. о представлении функции $q(z) \in L_{2}$, удовлетворяющей условиям

$$
\langle q(z), \varphi(z)\rangle=0, \quad \varphi(z) \in \mathscr{O}_{2}+\overline{\mathscr{O}}_{2}
$$

ТЕОРема 4.2. Функция $q(z) \in L_{2}$ удовлетворяет условиям (4.14) тогда и только тогда, когда существует (единственная) функиия $p(z) \in \stackrel{\circ}{W}{ }_{2}^{2}$ такая, что $q(z)=\Delta p(z)$ в области $G$. При этом

$$
\|p(z)\|_{W_{2}^{2}} \leqslant M\|q(z)\|_{0}
$$

где $M>0-$ постоянная.

ДокАЗАТЕЛЬСТвО. Достаточность немедленно следует из формулы $\Delta=4 \partial_{z} \partial_{\bar{z}}$ и предыдуших разложений. Обратно, если $q(z)$ удовлетворяет условиям (4.14), то последовательно решая задачи

$$
\begin{aligned}
\partial_{z} r(z) & =q(z), & \partial_{\bar{z}} p(z) & =r(z), \\
\left.r(z)\right|_{\Gamma} & =0, & \left.p(z)\right|_{\Gamma} & =0, \\
\langle q(z), \varphi(z)\rangle & =0, \quad \varphi(z) \in \mathscr{O}_{2}, & \langle r(z), \varphi(z)\rangle & =0, \quad \varphi(z) \in \overline{\mathscr{O}}_{2},
\end{aligned}
$$


получаем искомую функцию $p(z)$. При этом следует только заметить, что поскольку $r(z) \in \stackrel{\circ}{W} \underset{2}{1}(G)$, то в соответствии с замечанием 4.2 функция $p(z) \in \stackrel{\circ}{W}_{2}^{2}(G)$. Оценка (4.15) очевидна.

В заключение отметим, что вычисления показьвают, что

$$
p(z)=\frac{2}{\pi} \iint_{G} q(\zeta) \ln \frac{1}{|z-\zeta|} d \zeta d \eta
$$

где $\zeta=\xi+i \eta$. Иначе говоря, решение задачи

$$
\Delta p(z)=q(z),\left.\quad p(z)\right|_{\Gamma}=0,\left.\quad \frac{\partial}{\partial n} p(z)\right|_{\Gamma}=0
$$

сушествует в том и только в том случае, если функция $q(z) \in L_{2}$ ортогональна одновременно $\mathscr{O}_{2}$ и $\overline{\mathscr{O}}_{2}$ и выражается через стандартное фундаментальное решение оператора Лапласа.

4.1.3. Полная шкала аналитических соболевских пространств. Пусть $m \geqslant 1$ есть целое число и

$$
\mathscr{O}_{2}^{m}=\left\{f(z): f(z) \in \mathscr{O}_{2}, \ldots, f^{m}(z) \in \mathscr{O}_{2}\right\}
$$

есть пространство Соболева аналитических в области $G$ функций. Очевидно,

$$
\mathscr{O}_{2}^{m} \subset \mathscr{O}_{2}^{m-1} \subset \cdots \subset \mathscr{O}_{2}^{1} \subset L_{2}
$$

причем эти вложения плотны, ибо (в силу допустимости области определения $G$ ) в каждом пространстве $\mathscr{O}_{2}^{m}$ всюду плотно множество $B$ аналитических ограниченных в $G$ функций. Ясно также, что $\mathscr{O}_{2}^{m} \subset W_{2}^{m}$ и является замкнутым подпространством. Аналогично теореме 4.1 устанавливается

ТеОрема 4.3. Справедливы разложения:

$$
\begin{aligned}
& W_{2}^{m}=\mathscr{O}_{2}^{m}+\partial_{z}\left(W_{2}^{m+1} \cap \stackrel{\circ}{W}_{2}^{1}\right), \\
& W_{2}^{m}=\overline{\mathscr{O}}_{2}^{m}+\partial_{\bar{z}}\left(W_{2}^{m+1} \cap \stackrel{\circ}{W}_{2}^{1}\right), \\
& W_{2}^{m}=\mathscr{O}_{2}^{m}+\overline{\mathscr{O}}_{2}^{m}+\Delta\left(W_{2}^{m+1} \cap \stackrel{\circ}{W}_{2}^{1}\right) .
\end{aligned}
$$

4.1.4. Связь с $\bar{\partial}$-проблемой на плоскости. Как известно (см., например, $[9$, c. 51]), один из вариантов $\bar{\partial}$-проблемы состоит в нахождении условий на функции $q_{j}(z) \in \mathbb{C}_{0}^{m}\left(\mathbb{C}^{n}\right), j=1, \ldots, n(m \geqslant 1$ - целое число), при которых сушествует решение $f(z) \in \mathbb{C}_{0}^{m+1}\left(\mathbb{C}^{n}\right)$ системы Коши-Римана

$$
\partial_{\bar{z}_{j}} f(z)=q_{j}(z), \quad j=1, \ldots, n
$$

При $n>1$ ответ состоит в том, что правые части $f_{j}(z)$ должны удовлетворять естественным условиям согласования

$$
\partial_{\bar{z}_{k}} q_{j}(z) \equiv \partial_{\bar{z}_{j}} q_{k}(z), \quad k, j=1, \ldots, n .
$$


В предыдущем пункте получен ответ и для случая $n=1$, который, насколько нам известно, оставался открытым. Именно, $\bar{\partial}$-проблема на плоскости нормально разрешима в смысле Хаусдорфа, т.е. разрешима тогда и только тогда, когда правая часть $q(z) \in \stackrel{\circ}{W} \underset{2}{m}$ ортогональна антианалитическим функциям, т.е. ядру сопряженного оператора $\partial_{z}$. Решение при этом определяется единственным образом.

4.2. Конграничное и радиальное дифференцирование. Шкала пространств $W_{N, \Gamma}^{l, m}$.

4.2.1. Оператор конграничного ("углового") дифференцирования. При постановке нелинейной аналитической задачи (см. ниже п. 4.2.3) существенную роль играет специальный дифференциальный оператор первого порядка, который мы называем оператором конграничного дифференцирования. Этот же оператор играет определяюшую роль при описании аналитических функционалов над пространством Соболева аналитических функций.

Пусть $G$ - область с непрерьвно дифференцируемой границей $\Gamma$, звездная относительно начала координат. Определим на $\Gamma$ две функции $a(x, y)$ и $b(x, y)$, удовлетворяющие условиям

$$
\begin{gathered}
a(x, y)+i b(x, y)=1, \\
a(x, y) \cos \alpha+b(x, y) \sin \alpha=0,
\end{gathered}
$$

где $\alpha=\angle(n, x)$ - угол между внешней нормалью к гранище $\Gamma$ и осью $x$. Продолжим $a(x, y)$ и $b(x, y)$ внутрь области $G$ (исключая начало координат) как однородные функции и сохраним для этих продолжений те же обозначения. Ясно, что $a(x, y)$ и $b(x, y)-$ непрерьвные функции в $G \backslash\{0\}$.

ОПРЕДЕЛЕНИЕ 4.2. Оператором конграничного дифференцирования называется оператор

$$
\partial_{\Gamma} \equiv a(x, y) \frac{\partial}{\partial x}+b(x, y) \frac{\partial}{\partial y},
$$

где $a(x, y)$ и $b(x, y)$ - определенные выше функции.

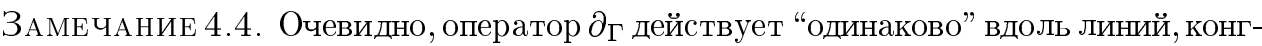
руэнтных границе $\Gamma$, чем и объясняется его название.

Отметим свойства оператора $\partial_{\Gamma}$.

УТВЕРЖДЕНИЕ 4.1. Оператор $\partial_{\Gamma}$ является расширением оператора аналитического дифференцирования, т.е. если $f(z) \in \mathscr{O}(G)$, то $\partial_{\Gamma} f(z) \equiv f^{\prime}(z)$.

Это утверждение есть прямое следствие условий Коши-Римана и условия (4.16).

УТВЕРЖДЕНИЕ 4.2. Пусть $\varphi(z) \in L_{2}, \partial_{\Gamma} \varphi(z) \in L_{2} ; f(z) \in L_{2}, \partial_{\Gamma}^{*} f(z) \in L_{2}$, əде

$$
\partial_{\Gamma}^{*} \circ=-\frac{\partial}{\partial x}(\bar{a}(z) \circ)-\frac{\partial}{\partial y}(\bar{b}(z) \circ)
$$

есть формально сопряжсенный оператор к $\partial_{\Gamma}$. Тогда

$$
\iint_{G} f(z) \overline{\partial_{\Gamma} \varphi(z)} d x d y=\iint_{G} \partial_{\Gamma}^{*} f(z) \overline{\varphi(z)} d x d y
$$

т.е. формально сопряженный оператор $\partial_{\Gamma}^{*}$ является и топологически сопряженным $к \partial_{\Gamma}$.

Данное утверждение является следствием условия (4.17). 
ЗАмЕчАНИЕ 4.5. В частности, если $\varphi(z)$ аналитична, то

$$
\iint_{G} f(z) \overline{\varphi^{\prime}(z)} d x d y=\iint_{G} \partial_{\Gamma}^{*} f(z) \overline{\varphi(z)} d x d y
$$

Легко устанавливается и

УТВЕРЖДЕНИЕ 4.3. Оператор

$$
J_{m}(f) \equiv \sum_{k=0}^{m}(-1)^{k}\left(\partial_{\Gamma}^{*}\right)^{k} f^{k}(z)
$$

является дуальным оператором пары $\mathscr{O}_{2}^{m}, \mathscr{O}_{2}^{-m}=\left(\mathscr{O}_{2}^{m}\right)^{*}(u$, в частности, отображсает изометрично $\mathscr{O}_{2}^{m}$ на $\left.\mathscr{O}_{2}^{-m}\right)$.

СлЕДСТВИЕ 4.1. Пространство $\mathscr{O}_{2}^{-m}$ состоит из тех и только тех аналитических функционалов, которые представимы в виде

$$
h(z) \equiv \sum_{k=0}^{m}\left(\partial_{\Gamma}^{*}\right)^{k} f^{k}(z),
$$

где $f_{k}(z) \in \mathscr{O}_{2}$ - некоторье функиии.

4.2.2. Оператор ортогонального ("радиального") дифференцирования. При построении оператора конграничного дифференцирования $\partial_{\Gamma}$ функции $a(x, y)$, $b(x, y)$ были продолжены внутрь области $G$ как однородные функции. Однако существует множество иных продолжений, сохраняюших утверждения 4.1-4.3 (при этом область $G$ может быть и незвездной). В частности, для построения продолжения функций $a(x, y), b(x, y)$, определенных на Г соотношениями $(4.16),(4.17)$, можно взять любое взаимно ортогональное семейство координатных линий, например, конформные образы полярной сетки единичного круга.

При наличии такого семейства можно построить дифференциальньй оператор $\partial_{N}$, “ортогональный” оператору $\partial_{\Gamma}$ (за последним сохраним название оператора конграничного дифференцирования). Именно, положим

$$
\partial_{N} \equiv c(x, y) \frac{\partial}{\partial x}+d(x, y) \frac{\partial}{\partial y}
$$

где функции $c(x, y)$ и $d(x, y)$ определяются из системы уравнений

$$
\begin{gathered}
c(x, y)+i d(x, y)=1, \\
\bar{a}(x, y) c(x, y)+\bar{b}(x, y) d(x, y)=0 .
\end{gathered}
$$

В силу (4.18) оператор $\partial_{N}$ на аналитических функциях совпадает с обычным комплексным дифференщированием (вдоль “радиальных" координатных линий).

Если через $\alpha=\alpha(x, y)$ обозначить угол между нормалью к “угловой” координатной линии в точке $(x, y)$ и осью ординат, то, как легко подсчитать,

$$
\begin{gathered}
\partial_{\Gamma}=i e^{-i \alpha} \sin \alpha \frac{\partial}{\partial x}-i e^{-i \alpha} \cos \alpha \frac{\partial}{\partial y} \\
\partial_{N}=e^{-i \alpha} \sin \alpha \frac{\partial}{\partial x}-e^{-i \alpha} \cos \alpha \frac{\partial}{\partial y} .
\end{gathered}
$$


При этом

$$
\frac{1}{2}\left(\partial_{\Gamma}+\partial_{N}\right)=\partial_{z}, \quad \frac{1}{2}\left(\partial_{\Gamma}^{*}+\partial_{N}^{*}\right)-\partial_{\bar{z}},
$$

где $\partial_{\Gamma}^{*}$ и $\partial_{N}^{*}-$ формально сопряженные операторы.

Таким образом, с любой ортогональной сеткой в области $G$ естественно связано дифференщиальное исчисление, порождаемое операторами углового и радиального дифференщирования, которые являются расширениями операторов аналитического дифференщирования. В частности, на формулы (4.21) можно смотреть как на "криволинейные" представления оператора Коши-Римана и сопряженного оператора.

4.2.3. Пример. Пусть $G=K$ - круг единичного радиуса. Тогда, как легко подсчитать, оператор $\partial_{\Gamma}$, соответствующий полярной сетке $(\rho, \theta)$, является с точностью до множителя $(i z)^{-1}$ оператором дифференцирования по полярному углу $\theta$, а именно,

$$
\partial_{\Gamma}=\frac{i y}{z} \frac{\partial}{\partial x}+\frac{-i x}{z} \frac{\partial}{\partial y}=\frac{1}{i z} \frac{\partial}{\partial \theta} .
$$

Будем назьвать этот оператор оператором комплексного углового дифферениирования и обозначать через $\partial_{\theta}$, т.е.

$$
\partial_{\theta}=\frac{i y}{z} \frac{\partial}{\partial x}+\frac{-i x}{z} \frac{\partial}{\partial y}=\frac{1}{i z} \frac{\partial}{\partial \theta} .
$$

Соответствуюший "ортогональньй” оператор $\partial_{N}$ (оператор радиального комплексного дифференцирования, которьй обозначим через $\partial_{\rho}$ ) определяется формулой

$$
\partial_{\rho}=\frac{x}{z} \frac{\partial}{\partial x}+\frac{y}{z} \frac{\partial}{\partial y} \equiv e^{-i \theta} \frac{\partial}{\partial \rho} .
$$

Справедливы формулы

$$
\partial_{\theta}+\partial_{\rho}=2 \partial_{z}, \quad \partial_{\theta}^{*}+\partial_{\rho}^{*}=-2 \partial_{\bar{z}}
$$

где $\partial_{\theta}^{*}, \partial_{\rho}^{*}$ - формально сопряженные операторы.

4.2.4. Пространства $W_{N, \Gamma}^{l, m}$. Операторы $\partial_{N}$ и $\partial_{\Gamma}$ позволяют ввести шкалу пространств типа пространств Соболева, которые, как нам представляется, весьма удобны при изучении аналитических задач вариационного типа.

Пусть $l \geqslant 0, m \geqslant 0$ - целые числа. Положим

$$
\begin{aligned}
W_{N, \Gamma}^{l, m}=\left\{r(z):\|r(z)\|_{l, m}^{2} \equiv\right. & \|r(z)\|_{L_{2}(G)}^{2} \\
& \left.+\sum_{k=1}^{l}\left\|\partial_{N}^{k} r(z)\right\|_{L_{2}(G)}^{2}+\sum_{k=1}^{l}\left\|\partial_{\Gamma}^{k} r(z)\right\|_{L_{2}(G)}^{2}<\infty\right\} .
\end{aligned}
$$

Очевидно (см. формулы (4.20)) для $l=1, m=1$ пространства $W_{N, \Gamma}^{1,1}$ совпадают с обычньми пространствами Соболева $W_{2}^{1}$. В других случаях это не так. 
Отметим, что пространства $W_{N, \Gamma}^{l, m}$ конформно эквивалентны в том смысле, что при невырожденном конформном отображении $w=w(z)$ области $G$ на единичньй круг $K$ пространство $W_{N, \Gamma}^{l, m}$ взаимно однозначно отображается на соответствующее пространство $W_{\rho, \theta}^{l, m}$ в круге $K$. При этом в силу формул

$$
\partial_{N}=w^{\prime}(z) \partial_{\rho}, \quad \partial_{\Gamma}=w^{\prime}(z) \partial_{\theta}
$$

норма пространства $W_{N, \Gamma}^{l, m}$ эквивалентна норме

$$
\|r(z)\|_{l, m}^{2} \equiv \sum_{k=1}^{l}\left\|\partial_{\rho}^{k} r(z)\right\|_{L_{2}(K)}^{2}+\sum_{k=0}^{m}\left\|\partial_{\theta}^{k} r(z)\right\|_{L_{2}(K)}^{2}
$$

соответствующего пространства $W_{\rho, \theta}^{l, m}$ в единичном круге.

В дальнейшем нам потребуются пространства $W_{N, \Gamma}^{0, m}$ и сопряженные к ним пространства $W_{N, \Gamma}^{0,-m}$. Ясно, что

$$
W_{N, \Gamma}^{0, m} \subset L_{2} \subset W_{N, \Gamma}^{0,-m}
$$

причем оператор

$$
\tilde{J} r(z) \equiv \sum_{k=0}^{m}\left(\partial_{\Gamma}^{*}\right)^{k}\left(\partial_{\Gamma}\right)^{k}
$$

является дуальным оператором пары $\left(W_{N, \Gamma}^{0, m}, W_{N, \Gamma}^{0,-m}\right)$. Тем самым всякий линейньй ограниченный функционал $q(z) \in W_{N, \Gamma}^{0,-m}$ представляется в виде

$$
q(z)=\sum_{k=0}^{m}\left(\partial_{\Gamma}^{*}\right)^{k} q_{k}(z)
$$

где $q_{k}(z) \in L_{2}(G)$ - некоторые функции.

Далее, поскольку оператор $\partial_{\Gamma}$ является расширением оператора комплексного дифференцирования, то $\mathscr{O}_{2}^{m} \subset W_{N, \Gamma}^{0, m}$ и, следовательно, $W_{N, \Gamma}^{0,-m} \subset \mathscr{O}_{2}^{-m}$. При этом оператор $\tilde{J} r(z)$ является расширением оператора

$$
J f(z) \equiv \sum_{k=0}^{m}\left(\partial_{\Gamma}^{*}\right)^{k} f^{(k)}(z): \mathscr{O}_{2}^{m}
$$

4.3. Основная нелинейная аналитическая задача. Теорема разрешимости.

4.3.1. Вновь "наводящий" модельный пример. Пусть $G$ - односвязная область с непрерывно дифференцируемой границей $\Gamma$. Как и в $\S 3$, в пространстве $\mathscr{O}_{2}^{1}$ рассмотрим задачу о минимизации функционала

$$
I(f)=\operatorname{Re} \iint_{G}\left[\frac{1}{2} a\left(z,\left|f^{\prime}(z)\right|^{2}\right)+\frac{1}{2} b\left(z,|f(z)|^{2}\right)-h(z) \bar{f}(z)\right] d x d y
$$


где $h(z) \in L_{2}$-некоторая функция, а функции $a(z, s), b(z, s)(z \in G, s \geqslant 0)$ непрерывны и удовлетворяют тем же неравенствам:

$$
\begin{gathered}
a_{0} s-k_{0} \leqslant \operatorname{Re} a(z, s) \leqslant A_{0} s+K_{0}, \\
b_{0} s-m_{0} \leqslant \operatorname{Re} b(z, s) \leqslant B_{0} s+M_{0}
\end{gathered}
$$

( $0<a_{0} \leqslant A_{0}<\infty, 0<b_{0} \leqslant B_{0}<\infty, k_{0}, K_{0}, m_{0}, M_{0}$ - некоторые постоянные $)$. Пусть, кроме того, функции $a(z, s), b(z, s)$ имеют ограниченные производные $a^{\prime}(z, s)$ и $b^{\prime}(z, s)$ по переменной $s$.

Как известно, в точке минимума

$$
\begin{aligned}
\delta I(f)(\varphi) \equiv & \operatorname{Re} \iint_{G}\left[a^{\prime}\left(z,\left|f^{\prime}(z)\right|^{2}\right) f^{\prime}(z) \overline{\varphi^{\prime}(z)}\right. \\
& \left.+b^{\prime}\left(z,|f(z)|^{2}\right) f(z) \overline{\varphi(z)}-h(z) \overline{\varphi(z)}\right] d x d y=0
\end{aligned}
$$

где $\varphi(z) \in \mathscr{O}_{2}^{1}-$ произвольная функция.

Вьведем соответствуюшее уравнение Эйлера. Для этого заметим, что, используя оператор конграничного дифференцирования $\partial_{\Gamma}$, равенство (4.22) можно представить в виде (см. утверждение 4.1)

$$
\iint_{G}\left[a^{\prime}\left(z,\left|f^{\prime}(z)\right|^{2}\right) f^{\prime}(z) \overline{\partial_{\Gamma} \varphi^{\prime}(z)}+b^{\prime}\left(z,|f(z)|^{2}\right) f(z) \overline{\varphi(z)}-h(z) \overline{\varphi(z)}\right] d x d y=0
$$

или (см. утверждение 4.2) в виде

$$
\iint_{G}\left[\partial_{\Gamma}^{*}\left(a^{\prime}\left(z,\left|f^{\prime}(z)\right|^{2}\right) f^{\prime}(z)\right)+b^{\prime}\left(z,|f(z)|^{2}\right) f(z)-h(z)\right] \overline{\varphi(z)} d x d y=0
$$

где $\varphi(z) \in \mathscr{O}_{2}^{1}$ (разумеется, мы должны здесь предположить, что на решении $f(z)$ функция

$$
\partial_{\Gamma}^{*}\left(a^{\prime}\left(z,\left|f^{\prime}(z)\right|^{2}\right) f^{\prime}(z)\right) \in L_{2}(G) .
$$

Далее, учитьвая, что вложение $\mathscr{O}_{2}^{1} \subset L_{2}$ всюду плотно (напомним, что $G$ - допустимая область), получаем, что последнее равенство справедливо для любой функции $\varphi(z) \in \mathscr{O}_{2}$. Следовательно, в силу основного разложения пространства $L_{2}(G)($ см. п. 4.1) найдется такая функция $r(z) \in \stackrel{\circ}{W} \frac{1}{2}(G)$, что

$$
\partial_{\Gamma}^{*}\left(a^{\prime}\left(z,\left|f^{\prime}(z)\right|^{2}\right) f^{\prime}(z)\right)+b^{\prime}\left(z,|f(z)|^{2}\right) f(z)+\partial_{z} r(z)=h(z)
$$

в смысле $L_{2}(G)$.

Это и есть уравнение Эйлера поставленной задачи минимизации.

Отметим его особенности:

1) как и ранее в $\S 3$, несмотря на то, что ставится задача отыскания аналитической функции, дающей минимум функционала, уравнение Эйлера существенно неаналитично, так как наряду с аналитическим дифференцированием $f^{\prime}(z)$ содержит и неаналитическое дифференцирование $\partial_{\Gamma}^{*}$, 
2) помимо функции $f(z) \in \mathscr{O}_{2}^{1}$ уравнение Эйлера содержит еще одну неизвестную функцию $r(z)$ (коаналитический потенциал), являющуюся "невязкой" между интегральньм и дифференциальньм представлениями функционального равенства $\delta I(f)(\varphi)=0$.

Завершая рассмотрение модельного примера, отметим, что, конечно, предположение (4.24) apriori неправомерно и поэтому в точке минимума $f(z) \in \mathscr{O}_{2}^{1}$ вариация

$$
\begin{aligned}
\delta I(f)(\varphi) \equiv & \operatorname{Re} \iint_{G}\left[a^{\prime}\left(z,\left|f^{\prime}(z)\right|^{2}\right) f^{\prime}(z) \partial_{\Gamma} \overline{\varphi(z)}\right. \\
& \left.+b^{\prime}\left(z,|f(z)|^{2}\right) f(z) \overline{\varphi(z)}-h(z) \overline{\varphi(z)}\right] d x d y
\end{aligned}
$$

определяет линейньй ограниченньй функционал только над $W_{N, \Gamma}^{0,1} \subset L_{2}$, которьй в силу (4.23) равен нулю на подпространстве $\mathscr{O}_{2}^{1}(G)$. Таким образом, уравнение Эйлера (4.25) имеет место в смысле теории обобшенных функций над пространством $W_{N, \Gamma}^{0,1}$, т.е. в смысле $W_{N, \Gamma}^{0,-1}$. Соответственно этому коаналитический потенщиал $r(z)$ должен быть таким, чтобы $\partial_{z} r(z) \in W_{N, \Gamma}^{0,-1}$.

Перейдем к строгим определениям и формулировкам.

4.3.2. Постановка задачи. Основная теорема. В области $G \subset \mathbb{C}^{1}$ с гранищей Г рассматривается нелинейная задача порядка $2 m$

$$
\begin{gathered}
L(f, r) \equiv \sum_{k=0}^{m}\left(\partial_{\Gamma}^{*}\right)^{k} A_{k}\left(z, f(z), \ldots, f^{(m)}(z)\right)+\partial_{z} r(z)=h(z) \\
\left.r(z)\right|_{\Gamma}=0
\end{gathered}
$$

или, что то же,

$$
\begin{gathered}
L(f, r) \equiv \sum_{k=0}^{m}\left(\partial_{\Gamma}^{*}\right)^{k} A_{k}\left(z, f(z), \ldots, f^{(m)}(z)\right)+\left(\partial_{N}+\partial_{\Gamma}\right) r(z)=h(z), \\
\left.r(z)\right|_{\Gamma}=0 .
\end{gathered}
$$

Здесь $A_{k}\left(z, \xi_{0}, \ldots, \xi_{m}\right)$ - функции, удовлетворяюшие условиям Каратеодори, т.е. измеримы и ограничены по $z \in G$ и непрерывны по $\xi_{0} \in \mathbb{C}^{1}, \ldots, \xi_{m} \in \mathbb{C}^{1}$.

Допустим, что вьполнены условия:

$$
\begin{aligned}
& \text { I. } \quad\left|A_{k}\left(z, \xi_{0}, \xi_{1}, \ldots, \xi_{m}\right)\right| \leqslant K\left(\sum_{k=0}^{m}\left|\xi_{k}\right|+1\right), \\
& \text { II. } \quad \operatorname{Re} \sum_{k=0}^{m} A_{k}\left(z, \xi_{0}, \ldots, \xi_{m}\right) \overline{\xi_{k}} \geqslant a_{0}\left(\left|\xi_{m}\right|^{2}+\left|\xi_{0}\right|^{2}\right)-K_{0},
\end{aligned}
$$

где $K>0, a_{0}>0, K_{0}>0$ - некоторые постоянные.

Решением поставленной задачи являются аналитическая функция $f(z) \in \mathscr{O}_{2}^{m}$ и неаналитическая функция $r(z) \in \nabla^{-1}\left(W_{N, \Gamma}^{0,-m}\right)$, где по определению

$$
\nabla^{-1}\left(W_{N, \Gamma}^{0,-m}\right)=\left\{r(z): r(z) \in W_{N, \Gamma}^{0,-m}, \partial_{\Gamma} r(z) \in W_{N, \Gamma}^{0,-m}, \partial_{N} r(z) \in W_{N, \Gamma}^{0,-m}\right\}
$$


(ниже мы поясним, что всякая функщия $r(z) \in \nabla^{-1}\left(W_{N, \Gamma}^{0,-m}\right)$ имеет след на границе $\Gamma$, так что граничное условие (4.27) (или (4.28)) имеет смысл).

Очевидно, при вьполнении условий I, II оператор $L(f, r)$ определен для любой пары $f(z) \in \mathscr{O}_{2}^{m}, r(z) \in \nabla^{-1}\left(W_{N, \Gamma}^{0,-m}\right)$, причем отображение

$$
L(f, r): \mathscr{O}_{2}^{m} \times \nabla^{-1}\left(W_{N, \Gamma}^{0,-m}\right) \rightarrow W_{N, \Gamma}^{0,-m}
$$

непрерывно.

Основньм результатом данного параграфа является

ТЕОРема 4.4. Пусть выполнены условия I, II. Тогда для любой правой части $h(z) \in W_{N, \Gamma}^{0,-m}$ существует по крайней мере одно решение $(f(z), r(z))$ задачи (4.28), (4.29) в том смысле, ито для любой основной функиии $\varphi(z) \in W_{N, \Gamma}^{0, m}$ справедливо тождество

$\sum_{k=0}^{m} \iint_{G} A_{k}\left(z, f(z), \ldots, f^{(m)}(z)\right) \overline{\partial_{\Gamma}^{k} \varphi(z)} d x d y+\left\langle\left(\partial_{N}+\partial_{\Gamma}\right) r(z), \varphi(z)\right\rangle=\langle h(z), \varphi(z)\rangle$

или, что то же,

$$
\sum_{k=0}^{m} \iint_{G} A_{k}\left(z, f(z), \ldots, f^{(m)}(z)\right) \overline{\partial_{\Gamma}^{k} \varphi(z)} d x d y+\left\langle\partial_{z} r(z), \varphi(z)\right\rangle=\langle h(z), \varphi(z)\rangle
$$

$\left(\langle h(z), \varphi(z)\rangle\right.$ - значение функиионала $h(z) \in W_{N, \Gamma}^{0,-m}$ на основной функиии $\varphi(z) \in$ $\left.W_{N, \Gamma}^{0, m}\right)$.

ДокАЗАТЕЛЬСТво. Доказательство проведем в два этапа: вначале методом компактности найдем функцию $f(z) \in \mathscr{O}_{2}^{m}$, а затем определим функцию $r(z)$ как решение эллиптической линейной задачи

$$
\partial_{N} r(z)+\partial_{\Gamma} r(z)=q(z),\left.\quad r(z)\right|_{\Gamma}=0,
$$

где

$$
q(z)=h(z)-\sum\left(\partial_{z}^{*}\right)^{k} A_{k}\left(z, f(z), \ldots, f^{(m)}(z)\right) \in W_{N, \Gamma}^{0,-m}
$$

есть возникшая "невязка".

1-й этап (определение $f(z))$. Пусть $\varphi_{1}(z), \varphi_{2}(z), \ldots$ - базисная система в пространстве $\mathscr{O}_{2}^{m} \equiv \mathscr{O}_{2}^{m}(G)$. Приближенное решение $f_{N}(z)$ ишем в виде

$$
f_{N}(z)=C_{1} \varphi_{1}(z)+\cdots+C_{N} \varphi_{N}(z),
$$

где постоянные $C_{1}, \ldots, C_{N}$ определяются из моментных уравнений Галеркина

$$
\begin{gathered}
\sum_{k=0}^{m} \iint_{G} A_{k}\left(z, f_{N}(z), \ldots, f_{N}^{(m)}(z)\right) \overline{\varphi_{j}^{(k)}(z)} d x d y=\left\langle h(z), \varphi_{j}(z)\right\rangle \\
j=1, \ldots, N
\end{gathered}
$$


(здесь учтено, что $\partial_{\Gamma}^{k} \varphi_{j}(z)=\varphi_{j}^{(k)}(z)$ ).

Разрешимость этой системы относительно $C_{1}, \ldots, C_{N}$ вытекает из условия коэрцитивности II и известной леммы "об остром угле". Из этого же условия для приближенных решений $f_{N}(z)$ следует оценка

$$
\sum_{k=0}^{m}\left\|f_{N}^{(k)}(z)\right\|_{L_{2}(G)} \leqslant K, \quad N=1,2, \ldots,
$$

где $K>0$ - постоянная, зависяшая от $h(z)$.

Действительно, умножая каждое равенство (4.30) на $\bar{C}_{j}$ и суммируя по $j=1, \ldots, N$, получаем, что

$$
\sum_{k=0}^{m} \iint_{G} A_{k}\left(z, f_{N}(z), \ldots, f_{N}^{(m)}(z)\right) \overline{f_{N}^{k}(z)} d x d y=\left\langle h, f_{N}\right\rangle
$$

или, с учетом условия II и вложения $\mathscr{O}_{2}^{m} \subset W_{N, \Gamma}^{0, m}$,

$$
a_{0} \sum_{k=0}^{m} \iint_{G}\left|f_{N}^{(k)}(z)\right|^{2} d x d y-A_{0} \leqslant\|h\|_{W_{N, \Gamma}^{0,-m}}\left\|f_{N}\right\|_{\mathscr{O}_{2}^{m}} .
$$

Отсюда немедленно и следует оценка (4.31). Из этой оценки и свойства монтелевости ограниченных множеств в $\mathscr{O}_{2}$ вытекает, что существуют функция $f(z) \in \mathscr{O}_{2}^{m}$ и такая подпоследовательность приближенных решений (обозначим ее вновь $f_{N}(z)$ ), что $f_{N}(z) \rightarrow f(z)$ локально равномерно в области $G$ со всеми производными. Переходя в $\left(4.30^{j}\right)$ к пределу при $N \rightarrow \infty$ (и принимая при этом во внимание условие I), находим, что

$$
\sum_{k=0}^{m} \iint_{G} A_{k}\left(z, f(z), \ldots, f^{(m)}(z)\right) \overline{\varphi_{j}^{(k)}(z)} d x d y=\left\langle h, \varphi_{j}(z)\right\rangle,
$$

где $j=1,2, \ldots$ произвольно.

Так как система $\varphi_{j}(z) \in \mathscr{O}_{2}^{m}, j=1,2, \ldots$, является полной, это означает, что

$$
\sum_{k=0}^{m} \iint_{G} A_{k}\left(z, f(z), \ldots, f^{(m)}(z)\right) \overline{\varphi^{(k)}(z)} d x d y=\langle h, \varphi(z)\rangle
$$

для любой функции $\varphi(z) \in \mathscr{O}_{2}^{m}$.

Итак, функция $f(z)$ найдена.

2-й этап (определение коаналитического потенщиала $r(z))$. Тождество (4.32) показывает, что разность

$$
q(z) \equiv h(z)-\sum_{k=0}^{m}\left(\partial_{\Gamma}^{*}\right)^{k} A_{k}\left(z, f(z), \ldots, f^{m}(z)\right),
$$

являюшаяся, очевидно, функционалом над $W_{N, \Gamma}^{0, m}$, обрашается в нуль на подпространстве аналитических функций $\mathscr{O}_{2}^{m}$. Таким образом, исходная задача будет решена, если мы докажем, что задача Дирихле

$$
\begin{gathered}
\partial_{N} r(z)+\partial_{\Gamma} r(z) \equiv q(z), \quad z \in G, \\
\left.r(z)\right|_{\Gamma}=0
\end{gathered}
$$

имеет (единственное) решение $r(z) \in \nabla^{-1}\left(W_{N, \Gamma}^{0,-m}\right)$. 
УТВЕРЖДЕНИЕ 4.4. Пусть $q(z) \in W_{N, \Gamma}^{0,-m}$, причем выполнено условие "ортогональности"

$$
\langle q(z), \varphi(z)\rangle=0, \quad \varphi(z) \in \mathscr{O}_{2}^{m}
$$

Тогда задача (4.33), (4.34) имеет единственное решение $r(z)$, причем

$$
\|r(z)\|_{-m}+\left\|\partial_{\Gamma} r(z)\right\|_{-m}+\left\|\partial_{N} r(z)\right\|_{-m} \leqslant M\|q(z)\|_{-m},
$$

где $\|\cdot\|_{-m}$ означает норму в $W_{N, \Gamma}^{0,-m}, M>0$ - постоянная.

ДокАЗАТЕЛЬСТво. Прежде всего, заметим, что задачу (4.33), (4.34) достаточно решить для единичного круга. Действительно, пусть $w=w(z): G \leftrightarrow K-$ невырожденное отображение области $G$ на единичный круг $K$. Как уже отмечалось, справедливы форомулы

$$
\partial_{N} r=w^{\prime}(z) \partial_{\rho} r, \quad \partial_{\Gamma} r=w^{\prime}(z) \partial_{\theta} r
$$

где $\partial_{\rho}$ и $\partial_{\theta}$ - операторы радиального и углового дифференцирования на плоскости $w=u+i v$. Следовательно, после замены переменных $w=w(z)$ задача (4.33), (4.34) преобразуется к виду

$$
\partial_{\rho} \tilde{r}(w)+\partial_{\theta} \tilde{r}(w)=\tilde{q}(w), \quad w \in K,\left.\quad \tilde{r}(w)\right|_{|w|=1}=0
$$

где $\tilde{r}(w)=r(z(w)), \tilde{q}(w)=q(z(w)) z^{\prime}(w)$, а $z(w)$ - отображение, обратное к $w(z)$.

Далее (это также отмечалось), из формул (4.37) вытекает, что в пространствах $W_{N, \Gamma}^{0, m}$ и $W_{\rho, \theta}^{0, m}$, а значит, и в сопряженных пространствах $W_{N, \Gamma}^{0,-m}$ и $W_{\rho, \theta}^{0,-m}$ нормы эквивалентны. Наконец, для любой функции $\varphi(w) \in \mathscr{O}_{2}^{m}(K)$

$$
\begin{aligned}
\langle\tilde{q}(w), \widetilde{\varphi}(w)\rangle & \equiv(\text { быть может, в смысле обобшенных функций }) \\
& \equiv \iint_{K} \tilde{q}(w) \overline{\widetilde{\varphi}(w)} d u d v=\iint_{G} q(z) \frac{\left|w^{\prime}(z)\right|^{2}}{w^{\prime}(z)} \overline{\varphi(z)} d x d y \\
& =\iint_{G} q(z) \overline{w^{\prime}(z) \varphi(z)} d x d y=0,
\end{aligned}
$$

т.е. условие ортогональности (4.35) также сохраняется.

Итак, без ограничения обшности можно считать, что $G$ есть единичный круг и задача (4.33), (4.34) имеет вид

$$
\begin{gathered}
\partial_{\rho} r(z)+\partial_{\theta} r(z)=q(z), \quad|z|<1 \\
\left.r(z)\right|_{|z|=1}=0
\end{gathered}
$$

где $(\rho, \theta)$ - полярные координаты на плоскости $z$. 
ЗАмечАнИЕ 4.6. Сейчас удобно пояснить в каком смысле выполняется граничное условие (4.39). Именно, пусть $r(z) \in \nabla^{-1}\left(W_{\rho, \theta}^{0,-m}\right)$. Это, в частности, означает, что

$$
\sup \frac{\left\langle\partial_{\rho} r(z), \varphi(z)\right\rangle}{\|\varphi(z)\|_{0, m}}<\infty
$$

(обозначения ясны). Рассмотрим функционал $r(z)=r(\rho, \theta)$ как функцию радиуса $\rho \in(0,1)$, принимающую значения в пространстве Соболева $W_{2}^{-m}(0,2 \pi)$ функций, периодических по угловой переменной $\theta \in(0,2 \pi)$, и положим $\varphi(z)=s(\rho) \phi(\theta)$, где $s(\rho) \in L_{2}(1 / 2,1), s(\rho) \equiv 0$ при $0<\rho<\frac{1}{2},\|s(\rho)\|_{L_{2}}=1 ; \phi(\theta) \in W_{2}^{m}(0,2 \pi)$, $\|\phi(\theta)\|_{m}=1$. Тогда из (4.40) следует, что

$$
\sup _{s, \phi} \int_{1 / 2}^{1} s(\rho) \frac{d}{d \rho}\langle r(\rho, \theta), \phi(\theta)\rangle d \rho<\infty
$$

т.е. функция $r(z) \equiv r(\cdot, \theta) \in W_{2}^{-m}(0,2 \pi)$ имеет производную

$$
\frac{d}{d \rho} r(\cdot, \theta) \in L_{2}\left(1 / 2,1 ; W_{2}^{-m}(0,2 \pi)\right)
$$

Следовательно, сама функций $r(\cdot, \theta) \in C\left(1 / 2,1 ; W_{2}^{-m}(0,2 \pi)\right)$ и тем самьм определено “сужение" $r(\rho, \cdot) \in W_{2}^{-m}(0,2 \pi)$ для каждого $\rho \in(1 / 2,1)$. В этом смысле и понимается условие (4.39).

Обратимся непосредственно к решению задачи (4.38), (4.39). Как в п. 4.1.1, формально имеем

$$
r(z)=\sum_{n=-\infty}^{+\infty} r_{n}(\rho) e^{i n \theta}
$$

где

$$
r_{n}(\rho)=\left\{\begin{array}{cc}
2 \int_{0}^{\rho} \eta^{n} q_{n-1}(\eta) d \eta \rho^{-n}, & n>0 \\
-2 \int_{\rho}^{1} \eta^{n} q_{n-1}(\eta) d \eta \rho^{-n}, & n \leqslant 0
\end{array}\right.
$$

$\left(q_{n-1}(\rho)-\right.$ соответствующая проекция $q(z)$ в разложении в ряд Фурье). Ясно, что, доказав оценку (4.36), мы докажем и утверждение.

Вывод оценки (4.36). Прежде всего, заметим, что, используя дуальное отображение $\tilde{J} q(z): W_{\rho, \theta}^{0, m} \rightarrow W_{\rho, \theta}^{0,-m}$, нетрудно вычислить норму $\|q(z)\|_{-m}$ в пространстве $W_{\rho, \theta}^{0,-m}$, а именно,

$$
\|q(z)\|_{-m}^{2}=\frac{1}{2 \pi} \sum_{n=-\infty}^{\infty} \int_{0}^{1} \frac{\rho^{2 m+1}}{s_{m n}(\rho)}\left|q_{n}(\rho)\right|^{2} d \rho
$$

где

$$
s_{m n}(\rho)=\rho^{2 m}+\sum_{k=1}^{m} n^{2}(n-1)^{2} \cdots(n-k+1)^{2} \rho^{2(m-k)} .
$$


Далее, поскольку

$$
\partial_{\theta} r(z)=\sum_{n=-\infty}^{\infty} \frac{n+1}{\rho} r_{n+1}(\rho) e^{i n \theta}
$$

имеем

$$
\left\|\partial_{\theta} r(z)\right\|_{-m}^{2}=\frac{1}{2 \pi} \sum_{n=-\infty}^{\infty}(n+1)^{2} \int_{0}^{1} \frac{\rho^{2 m-1}}{s_{m n}(\rho)}\left|r_{n+1}(\rho)\right|^{2} d \rho
$$

и это выражение надо оценить через (4.42).

Соответствуюшую оценку получим для различных груп значений $n$. При этом будем сушественно использовать классические неравенства Харди (см., например, $[10],[11]):$
a) $\int_{0}^{1} \rho^{s}\left|\int_{0}^{\rho} f(\eta) d \eta\right|^{2} d \rho \leqslant \frac{4}{(s+1)^{2}} \int_{0}^{1} \rho^{s+2}|f(\rho)|^{2} d \rho \quad(s<-1)$;
б) $\int_{0}^{1} \rho^{s}\left|\int_{\rho}^{1} f(\eta) d \eta\right|^{2} d \rho \leqslant \frac{4}{(s+1)^{2}} \int_{0}^{1} \rho^{s+2}|f(\rho)|^{2} d \rho \quad(s>-1)$.

I. Пусть $n=0,1, \ldots, m-1$. Тогда (при $\rho \rightarrow+0) s_{m n}^{-1}(\rho) \approx \rho^{2(n-m)}$ и дело сводится к неравенству

$$
\int_{0}^{1} \rho^{2 n-1}\left|r_{n+1}\right|^{2} d \rho \leqslant M \int_{0}^{1} \rho^{2 n+1}\left|q_{n}(\rho)\right|^{2} d \rho
$$

где $M>0-$ постоянная.

Имеем:

$$
\begin{aligned}
& \int_{0}^{1} \rho^{2 n-1}\left|r_{n+1}(\rho)\right|^{2} d \rho=4 \int_{0}^{1} \rho^{2 n-1}\left|\int_{0}^{\rho} \eta^{n+1} q_{n}(\eta) d \eta\right|^{2} \rho^{-2(n+1)} d \rho \\
& \left.\quad=4 \int_{0}^{1} \rho^{-3}\left|\int_{0}^{\rho} \eta^{n+1} q_{n}(\eta) d \eta\right|^{2} d \rho \leqslant(\text { неравенство Харди а })\right) \\
& \quad \leqslant M \int_{0}^{1} \rho^{2 n+1}\left|q_{n}(\rho)\right|^{2} d \rho \quad(M>0),
\end{aligned}
$$

что и требуется.

II. Пусть теперь $n \neq 0,1, \ldots, m-1$. Тогда (при $|n| \rightarrow \infty) s_{m n}(\rho) \approx n^{2}(n-1)^{2} \ldots$ $\cdots(n-m+1)^{2}$ и дело сводится к оценке

$$
(n+1)^{2} \int_{0}^{1} \rho^{2 m-1}\left|r_{n+1}(\rho)\right|^{2} d \rho \leqslant M \int_{0}^{1} \rho^{2 m+1}\left|q_{n}(\rho)\right|^{2} d \rho .
$$

Последняя оценка устанавливается вполне аналогично п. I, при этом в случае $n \geqslant m$ используется неравенство Харди а), а в случае $n<0-$ неравенство Харди б). (В обоих случаях при этом $s=2(m-n)-3$.)

Ясно, что из равенства Парсеваля теперь получаем, что

$$
\left\|\partial_{\theta} r(z)\right\|_{0,-m} \leqslant M\|q(z)\|_{0,-m}
$$


Аналогично (и даже чуть легче) получается неравенство

$$
\|r(z)\|_{0,-m} \leqslant M\|q(z)\|_{0,-m}
$$

Что же касается неравенства

$$
\|\partial \rho r(z)\|_{0,-m} \leqslant M\|q(z)\|_{0,-m}
$$

то оно немедленно вытекает из уравнения (4.38).

Тем самым искомая оценка (4.36) установлена.

Это завершает доказательство утверждения 4.4, а вместе с ним, как уже отмечалось, и доказательство основной теоремы 4.4 .

ЗАмЕчАниЕ 4.7. Если выполнено условие строгой монотонности, то решение задачи (4.28), (4.29) единственно.

ПримеР. Рассмотрим уравнение в круге

$$
\frac{\partial}{\partial x}\left(\frac{i y}{\bar{z}} f^{\prime}(z)\right)-\frac{\partial}{\partial y}\left(\frac{i x}{\bar{z}} f^{\prime}(z)\right)+f(z)+\partial_{z} r(z)=h(z)
$$

(уравнение Эйлера функционала $\left.\left\|f^{\prime}\right\|_{0}^{2}+\|f\|_{0}^{2}-\langle h, f\rangle\right)$. Это линейное уравнение с ограниченными измеримыми коэффициентами. Условия I, II вьполнены. Этому уравнению можно придать вид

$$
\partial \theta^{*} \partial \theta f(z)+f(z)+(\partial \rho+\partial \theta) r(z)=h(z) .
$$

Интерес этого примера в том, что (в отличие от рассмотренного в $\S 3$ периодического случая) в данной вариационной задаче уравнение Эйлера содержит коаналитический потенциал $r(z)$ даже в том случае, когда правая часть $h(z)$ аналитична.

4.3.3. Заключительные замечания. Вьше детально рассмотрена аналитическая задача, отвечающая разложению

$$
L_{2}=\mathscr{O}_{2}+\partial_{z} \stackrel{\circ}{W} \underset{2}{1}
$$

Совершенно аналогично изучается случай, соответствуюший "антианалитическому" разложению

$$
L_{2}=\overline{\mathscr{O}}_{2}+\partial_{\bar{z}} \stackrel{\circ}{W}_{2}^{1}
$$

В этом случае соответствующая задача имеет вид

$$
\sum_{k=0}^{m}\left(\delta_{\Gamma}^{*}\right) A_{k}\left(\bar{z}, f_{\bar{z}}(\bar{z}), \ldots, f_{\bar{z}}^{(m)}(\bar{z})\right)+\partial_{\bar{z}} r(\bar{z})=h(\bar{z}),\left.\quad r(\bar{z})\right|_{\Gamma}=0
$$

где роль оператора конграничного дифференцирования играет оператор $\delta_{\Gamma}$, получающийся из оператора $\partial_{\Gamma}$ заменой $y$ на $-y$, т.е.

$$
\delta_{\Gamma}=a(x,-y) \frac{\partial}{\partial x}-b(x,-y) \frac{\partial}{\partial y} .
$$


Ясно теперь, что нелинейная задача, отвечающая "гармоническому" разложению

$$
L_{2}=\mathscr{O}_{2}+\overline{\mathscr{O}}_{2}+\partial_{z} \partial_{\bar{z}} \stackrel{\circ}{W}_{2}^{2}
$$

т.е. задача вариационного типа на подпространстве гармонических функций, представляется в виде

$$
\sum_{k+l \leqslant m}\left(\partial_{\Gamma}^{*}\right)^{k}\left(\delta_{\Gamma}^{*}\right)^{l} A_{k l}\left(z, \bar{z}, \mathscr{D}_{z \bar{z}}^{\gamma} f(z, \bar{z})\right)+\Delta p(z, \bar{z})=h(z, \bar{z}), \quad|\gamma| \leqslant m .
$$

При этом никакие граничные условия на гармоническую функцию $f(z, \bar{z})$ не накладываются. Напротив, когармонический потенциал $p(z, \bar{z})$ обрашается на гранище в нуль вместе со всеми производными первого порядка.

\section{$\S 5$. Комментарии и задачи}

Теорема 1.1 об аналитическом продолжении решений обькновенных дифференщиальных уравнений относилась к случаю классических решений уравнения

$$
f_{0}^{(n)}(x)+\sum_{k=0}^{n-1} a_{k}(x) f_{0}^{(k)}(x)=h(x), \quad x \in(a, b),
$$

т.е. к случаю $f_{0}(x) \in \mathbb{C}^{n}(a, b)$ при непрерывных коэффициентах и правых частях уравнения.

ЗАДАЧА 1. Исследовать аналогичную задачу об аналитическом продолжении решения уравнения (5.1) в случаях: а) $f_{0}(x) \in W^{n}(a, b)$ - функиия из пространства Соболева; б) $f_{0}(x) \in \mathscr{D}(a, b)$ - обобщенная функиия; в) $f_{0}(x)$ - гиперфункиия.

Интерес представляют достаточные условия на коэффициенты уравнения, обеспечиваюшие разрешимость многоточечных задач, отличных от задач, рассмотренных в $§ 2$. Примером такой задачи является, например,

ЗАДАЧА 2. Указать условия на а(z), достаточные для разрешимости задачи

$$
\begin{gathered}
f^{(\mathrm{iv})}(z)+a(z) f(z)=h(z), \quad z \in G, \\
f\left(z_{1}\right)=f_{01}, \quad f^{\prime}\left(z_{1}\right)=f_{11}, \quad f\left(z_{2}\right)=f_{02}, \quad f\left(z_{3}\right)=f_{03},
\end{gathered}
$$

әде $z_{1}, z_{2}, z_{3}$ - фиксированные точки в области $G$.

Отметим, что вне рамок данной статьи остались нелокальные многоточечные задачи.

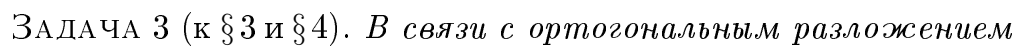

$$
L_{2}=\mathscr{O}_{2} \dot{+} \partial_{z} \stackrel{\circ}{W}_{2}^{1,1}
$$

и соответствующим разложением $f(z) \in L_{2}$ на аналитическую и коаналитическую составляющие (см. §3) выяснить возможный физический смысл коаналитического потенциала $r(z)$. То же самое и по отношению к разложениям $(c \mathcal{M} . \S 4)$

$$
\begin{gathered}
L_{2}=\mathscr{O}_{2}+\partial_{z} \stackrel{\circ}{W_{2}^{1,1}}, \quad L_{2}=\overline{\mathscr{O}}_{2}+\partial_{\bar{z}} \stackrel{\circ}{W}_{2}^{1,1}, \\
L_{2}=\mathscr{O}_{2} \dot{+} \overline{\mathscr{O}}_{2} \dot{+}+\stackrel{\circ}{W}_{2}^{2} .
\end{gathered}
$$


ЗАДАЧА 4. Пусть $\varphi_{1}(x), \ldots, \varphi_{n}(x), \ldots$ - вещественно-аналитический базис в $L_{2}(a, b)$, әде $-\infty \leqslant a<b \leqslant+\infty$. В каком случае этот базис аналитически продолжсатся в область $G$ как базис в пространстве $\mathscr{O}_{2}(G)$, где $G \subset \mathbb{C}_{z}^{1}$ - некоторая область, содержащая $(a, b)$. Рассмотреть примеры классических базисов и выяснить их области продолжсения как базисов в $\mathscr{O}_{2}(G)$.

ЗАДАчА 5. Разработать $L_{p}$-теорию нелинейньх аналитических задач из $§ 3 и$ $\S 4$.

В связи с понятием допустимой области (см. $\S 4)$ возникает вопрос о справедливости теоремы Рунге в пространстве $\mathscr{O}_{2}(G)$.

ЗАДАчА 6 . Пусть $G$ - ограниченная односвязная область в $\mathbb{C}_{z}^{1}$. Тогда, какова бы ни была функция $f(z) \in \mathscr{O}_{2}$, существует последовательность многочленов $P_{n}(z), n=1,2, \ldots$, сходящаяся в пространстве $\mathscr{O}_{2} \kappa$ функции $f(z)$. Верно ли эmo?

Пусть $G$ - односвязная область с гладкой границей $\Gamma$; далее, $\partial_{\Gamma}$ и $\partial_{N}-$ операторы конграничного и радиального дифференцирования (см. $\S 4$, п. 4.2.1).

ЗАДАчА 7. Построить теорию задачи Дирихле для уравнения

$$
\partial_{\Gamma} r(z)+\partial_{N} r(z)=q(z), \quad z \in G,
$$

в полной шкале пространств $W_{N, \Gamma}^{l, m}$.

ЗАДАЧА 8. То же - для задачи Дирихле для уравнения Пуассона

$$
\Delta u=h(z), \quad z \in G,
$$

а также для переопределенной задачи

$$
\Delta u=h(z),\left.\quad u\right|_{\Gamma}=0,\left.\quad \frac{\partial u}{\partial n}\right|_{\Gamma}=0
$$

Большой блок интересных, на мой взгляд, вопросов возникает при обобшении рассмотренных задач на случай многих переменных и, прежде всего, при попытках осмыслить проблему аналитического продолжения решений краевых задач "вешественной” математической физики.

С этой целью заметим, что метод продолжения решений обыкновенных дифференциальных уравнений, рассмотренньй в $\S 1$, формально пригоден и для продолжения решений уравнений с частными производными. Поясним это, например, для уравнения теплопроводности. Пусть $u_{0}(t, x)$ - решение уравнения

$$
\frac{\partial u_{0}}{\partial t}-a(t, x) \frac{\partial^{2} u_{0}}{\partial x^{2}}=h(t, x)
$$

определенное при $t \in\left(t_{1}, t_{2}\right), x \in\left(x_{1}, x_{2}\right)$.

Продолжим вначале это решение по переменной $z=x+i y$ как решение уравнения

$$
\begin{gathered}
\frac{\partial u}{\partial t}(t, z)-a(t, z) \frac{\partial^{2} u}{\partial z^{2}}(t, z)=h(t, z), \\
t \in\left(t_{1}, t_{2}\right), \quad(x, y) \in\left(x_{1}, x_{2}\right) \times\left(y_{-}, y_{+}\right), \quad y_{-} \leqslant 0, \quad 0<y_{+},
\end{gathered}
$$


считая $a(t, z)$ и $h(t, z)$ аналитическими продолжениями $a(t, x)$ и $h(t, x)$ в область $\left(x_{1}, x_{2}\right) \times\left(y_{-}, y_{+}\right)$.

Для этого определим $u(t, z), z=x+i y$, как решение задачи Коши по переменной $y$, а именно (см. доказательство теоремы 1.1)

$$
\begin{aligned}
\frac{\partial u}{\partial t}+\frac{\partial^{2} u}{\partial y^{2}} & =h(t, z), \quad t \in\left(t_{1}, t_{2}\right), \quad y \in\left(y_{1}, y_{2}\right), \\
u(t, x+i 0) & =u_{0}(t, x), \quad \frac{\partial u}{\partial y}(t, x+i 0)=i \frac{\partial u_{0}}{\partial x}(t, x)
\end{aligned}
$$

$\left(x \in\left(x_{1}, x_{2}\right)\right.$ - параметр).

Ясно, что так определенная функция $u(t, z)$ будет аналитической по $z$, ибо функция $w(t, z) \equiv \partial_{\bar{z}} u(t, z)$ является решением однородной задачи Коши

$$
\begin{gathered}
\frac{\partial w}{\partial t}(t, z)+\frac{\partial^{2} w}{\partial y^{2}}(t, z)=0 \\
w(t, x+i 0)=0, \quad \frac{\partial w}{\partial y}(t, x+i 0)=0
\end{gathered}
$$

Далее, найденная функция $u(t, z)$ аналогичным образом продолжается аналитически по переменной $t$. В итоге получаем решение $u(s, z), s=t+i \sigma$, комплексного "уравнения теплопроводности"

$$
\frac{\partial u}{\partial s}-\frac{\partial^{2} u}{\partial z^{2}}=h(s, z)
$$

такое, что $u(t+i 0, x+i 0)=u_{0}(t, x)$. Это и требовалось.

Отметим также, что если решение $u_{0}(t, z)$ определено на всей вешественной оси, то проблему его продолжения по $z$ можно рассматривать как проблему продолжения вешественной задачи Коши

$$
\begin{gathered}
\frac{\partial u_{0}}{\partial t}-a(t, x) \frac{\partial^{2} u_{0}}{\partial x^{2}}=h(t, x), \\
u_{0}(0, x)=\varphi_{0}(x), \quad t \in[0, T), \quad x \in \mathbb{R}^{1} .
\end{gathered}
$$

В этом случае возможен другой вариант продолжения (возможно, более простой). Именно, пусть $a(t, z), h(t, z)$ и $\varphi_{0}(z)$ - аналитические продолжения $a(t, x), h(t, x)$ и $\varphi_{0}(x)$, соответственно. Рассмотрим задачу Коши

$$
\frac{\partial u}{\partial t}-a(t, z) \frac{\partial^{2} u}{\partial x^{2}}(t, z)=h(t, z), \quad u_{0}(0, z)=\varphi_{0}(z)
$$

где, в отличие от предыдущего примера, в качестве параметра выступает $y \in\left(y_{-}, y_{+}\right)$. Понятно, что и в этом случае функция $w \equiv \partial_{\bar{z}} u$ удовлетворяет однородной задаче

$$
\frac{\partial w}{\partial t}-a(t, z) \frac{\partial^{2} w}{\partial x^{2}}=0, \quad w_{0}(0, z)=0
$$


т.е. найденное решение $u(t, z)$ формально аналитично.

Очевидно, обоснование данных приемов аналитического продолжения упирается, как минимум, в следующие проблемы.

1. Разрешимость задачи Коши в пространствах вешественно-аналитических функций для уравнений с частными производными, зависящими от параметра.

При этом необходимо иметь теоремы об аналитической (или конечно-гладкой) зависимости решения от параметра - аналог классической теоремы Пуанкаре-Перрона для обыкновенного дифференциального уравнения.

2. Разрешимость задачи Коши для уравнений, у которых часть независимых переменных вешественна, а часть комплексна.

Следует подчеркнуть при этом, что соответствуюшие уравнения не являются, вообще говоря, уравнениями типа Ковалевской, поэтому речь может идти в этих случаях о разрешимости задачи Коши только в определенных подпространствах аналитических функций, например, экспоненциальных функций, аналитических периодических функций и др. (напомним, что локальная корректность задачи Коши в классе всех аналитических функций имеет место только для уравнения типа Ковалевской).

Понятно, что решение этих проблем для каждого индивидуального уравнения будет требовать и индивидуального подхода. Хотелось бы осмыслить все это прежде всего для трех классических типов уравнений математической физики.

\section{§. Об одной задаче продолжения с наименьшим коаналитическим уклонением}

В этом параграфе формулируется математическая модель задачи о продолжении внутрь области граничной функции, наименее уклоняющемся от подпространства аналитических функций (имеющем наименьшее коаналитическое уклонение). Устанавливается корректность поставленной задачи. В частности, для круга таким продолжением является гармоническое продолжение.

6.1. Постановка задачи.

Пусть $G \subset \mathbb{C}^{1}(z=x+i y)$ - область с границей Г. Как и ранее, обозначим через $L_{2}(G)$ пространство Лебега функций $f(z): G \rightarrow \mathbb{C}^{1}$, суммируемых с квадратом в области $G$, а через $\mathscr{O}_{2}(G)$ - замкнутое в нем подпространство аналитических функций, т.е.

$$
\mathscr{O}_{2}(G)=\left\{f(z): f(z) \in L_{2}(G), \partial_{\bar{z}} f(z) \equiv 0\right\},
$$

где $\partial_{\bar{z}}$ - оператор Коши-Римана. Тогда (см. $\S 3$ имеет место ортогональное разложение

$$
L_{2}(G)=\mathscr{O}_{2}(G) \oplus \partial_{z} \stackrel{\circ}{W} \underset{2}{1}(G)
$$

где $\partial_{z}$ - оператор, сопряженньй к оператору Коши-Римана, а $\stackrel{\circ}{W} \underset{2}{1}(G)$ - пространство Соболева функций, обращаюшихся в нуль на гранище Г. Это значит, что всякая функция $f(z) \in L_{2}(G)$ однозначно представляется в виде суммы $f(z)=f_{a}(z)+f_{c a}(z)$, где $f_{a}(z) \in \mathscr{O}_{2}(G)$ - ее аналитическая часть, а $f_{c a}(z) \in \mathscr{O}_{2}^{\perp}(G)$ - ее коаналитическая часть; при этом существует единственная функция $r(z) \in \stackrel{\circ}{W} \underset{2}{1}(G)$ (коаналитический потенциал функции $f(z))$ такая, что $f_{c a}(z)=\partial_{z} r(z)$.

Очевидно, функция $f_{c a}(z)$ может служить определенной характеристикой уклонения самой функции $f(z)$ от подпространства аналитических функщий. 
ОПРЕДЕЛЕНИЕ. Пусть $f(z) \in W_{2}^{1}(G)$. Мерой коаналитичности (или коаналитическим уклонением) функции $f(z)$ назьвается число

$$
\mu(f)=\left\|f-f_{a}\right\|_{W_{2}^{1}(G)}^{2},
$$

то есть

$$
\mu(f)=\iint_{G}\left[\left|\nabla\left(f-f_{a}\right)\right|^{2}+\left|f-f_{a}\right|^{2}\right] d x d y
$$

В связи с этим определением возникает задача. Пусть $f_{0}(\gamma) \in W_{2}^{1 / 2}(\Gamma)$ - некоторая граничная функция. Среди всевозможных ее продолжений $f(z) \in W_{2}^{1}(G)$ найти то продолжение, которое имеет наименьшее коаналитическое уклонение. Иными словами, требуется найти продолжение заданной граничной функции, наименее уклоняющееся от подпространства аналитических функций в смысле характеристики $\mu(f)$.

Очевидно, эта задача есть задача минимизации функционала $\mu(f)$ на подмножестве функций $f(z) \in W_{2}^{1}(G)$ таких, что $\left.f(z)\right|_{\Gamma}=f_{0}(\gamma), \gamma \in \Gamma$.

\section{2. Математическая модель задачи продолжения.}

Выведем математическую модель поставленной задачи, считая временно, что решение $f(z) \in W_{2}^{2}(G)$, т.е. имеет на единищу большую гладкость.

В соответствии с необходимым условием экстремума имеем для любой функции $\varphi(z) \in \stackrel{\circ}{W} \frac{1}{2}(G)$

$$
\delta \mu(f)(\varphi) \equiv \iint_{G}\left[\nabla\left(f-f_{a}\right) \nabla\left(\bar{\varphi}-\bar{\varphi}_{a}\right)+\left(f-f_{a}\right)\left(\bar{\varphi}-\bar{\varphi}_{a}\right)\right] d x d y=0
$$

откуда, положив $\varphi(z)=\partial_{z} r(z)$, где $r(z) \in \mathscr{D}(G)$, находим, что

$$
\iint_{G}\left[-\Delta\left(f-f_{a}\right)+\left(f-f_{a}\right)\right] \bar{\varphi}(z) d x d y=0
$$

ибо для указанных функций $\varphi_{a}(z)=0$. Отсюда немедленно находим, что искомое продолжение $f(z)$ удовлетворяет включению

$$
-\Delta\left(f-f_{a}\right)+\left(f-f_{a}\right) \in \mathscr{O}_{2}(G)
$$

или, что то же, включению

$$
-\Delta f(z)+f(z) \in \mathscr{O}_{2}(G)
$$

Обратимся к выводу граничного условия.

С этой целью вновь рассмотрим тождество (6.1), полагая в нем произвольно $\varphi(z) \in$ $\stackrel{\circ}{W} \underset{2}{1}(G)$. Тогда, интегрируя по частям, находим, что

$$
\left\langle-\Delta\left(f-f_{a}\right)+f-f_{a}, \varphi-\varphi_{a}\right\rangle_{G}+\left.\int_{\Gamma} \frac{\partial}{\partial n}\left(f-f_{a}\right) \bar{\varphi}_{a}(z)\right|_{\Gamma} d \gamma=0
$$


( $\vec{n}$ - направление внешней нормали к Г) или, учитьвая включение (6.2),

$$
\left.\int_{\Gamma} \frac{\partial}{\partial n}\left(f-f_{a}\right) \bar{\varphi}_{a}(z)\right|_{\Gamma} d \gamma=0 .
$$

Обозначим через $\mathscr{O}_{2}^{1 / 2}(\Gamma)$ множество граничных значений аналитических составляюших функций $\varphi(z) \in \stackrel{\circ}{W} \underset{2}{1}(G)$, т.е.

$$
\mathscr{O}_{2}^{1 / 2}(\Gamma)=\left\{\varphi_{a}(\gamma): \varphi_{a}(\gamma)=\left.\varphi_{a}(z)\right|_{\Gamma}, \varphi(z) \in \stackrel{\circ}{W}{ }_{2}^{1}(G)\right\}
$$

Очевидно, $\mathscr{O}_{2}^{1 / 2}(\Gamma) \subset W_{2}^{1 / 2}(\Gamma)$ и является замкнутым подпространством пространства $W_{2}^{1 / 2}(\Gamma)$. Следовательно, обозначив через $P_{a}$ проектор

$$
P_{a}: W_{2}^{1 / 2}(\Gamma) \rightarrow \mathscr{O}_{2}^{1 / 2}(\Gamma),
$$

условие (6.3) можно записать в виде

$$
P_{a}\left\{\left.\frac{\partial}{\partial n}\left(f-f_{a}\right)\right|_{\Gamma}\right\}=0 .
$$

Таким образом, решение $f(z)$ поставленной задачи минимизации удовлетворяет дифференщиальному включению

$$
-\Delta f(z)+f(z) \in \mathscr{O}_{2}(G)
$$

и двум граничным условиям

$$
\begin{gathered}
\left.f(z)\right|_{\Gamma}=f_{0}(\gamma), \\
P_{a}\left\{\left.\frac{\partial}{\partial n}\left(f-f_{a}\right)\right|_{\Gamma}\right\}=0,
\end{gathered}
$$

последнее из которых носит проекционньй характер.

Это и есть искомая математическая модель.

Напомним, однако, что эта модель вьведена нами при дополнительном условии $f(z) \in W_{2}^{2}(G)$. В общем случае этого включения, конечно, может не быть, и, строго говоря,

$$
L f \equiv-\Delta\left(f-f_{a}\right)+f-f_{a} \in W_{2}^{-1}(G),
$$

т.е. является линейным непрерывным функционалом над пространством $\stackrel{\circ}{W} \underset{2}{1}(G)$. Этот функционал можно, очевидно, представить в виде суммы аналитического и коаналитического функционалов

$$
L(f)=L_{a}(f)+L_{c a}(f),
$$

которые действуют по формулам

$$
\begin{gathered}
L_{a}(f) \varphi_{a}=\left\langle\nabla\left(f-f_{a}\right)+\left(f-f_{a}\right), \varphi_{a}\right\rangle \\
L_{c a}(f)\left(\varphi-\varphi_{a}\right)=\left\langle\nabla\left(f-f_{a}\right), \nabla\left(\varphi-\varphi_{a}\right)\right\rangle+\left\langle f-f_{a}, \varphi-\varphi_{a}\right\rangle .
\end{gathered}
$$


Поскольку $L_{c a}(f)=0$, то $L(f)=L_{a}(f)$, т.е. $L(f)$ можно отождествить с функционалом $L_{a}(f)$ над подпространством аналитических функций

$$
\mathscr{O}_{2}^{1}(G)=\left\{f(z) \in W_{2}^{1}(G), \partial_{\bar{z}} f(z) \equiv 0\right\} .
$$

Будем обозначать сказанное включением $L(f) \in \mathscr{O}_{2}^{-1}(G)$.

В конечном итоге, математическая модель задачи о продолжении граничной функции $f_{0}(\gamma) \in W_{2}^{1 / 2}(\Gamma)$, имеюшем наименьшее коаналитическое уклонение, принимает вид

$$
\begin{gathered}
-\Delta\left(f-f_{a}\right)+\left(f-f_{a}\right) \in \mathscr{O}_{2}^{-1}(G), \\
\left.f(z)\right|_{\Gamma}=f_{0}(\gamma), \\
P_{a}\left\{\left.\frac{\partial}{\partial n}\left(f-f_{a}\right)\right|_{\Gamma}\right\}=0
\end{gathered}
$$

или, что то же,

$$
\begin{gathered}
-\Delta f+f \in \mathscr{O}_{2}^{-1}(G), \\
\left.f(z)\right|_{\Gamma}=f_{0}(\gamma), \\
P_{a}\left\{\left.\frac{\partial}{\partial n}\left(f-f_{a}\right)\right|_{\Gamma}\right\}=0 .
\end{gathered}
$$

При этом последнее условие понимается в смысле теории следов обобщенных функций со значениями в $W_{2}^{-1}(\Gamma)$.

\section{3. Теорема существования и единственности.}

Теорема 6.1. Для любой функиии $f_{0}(\gamma) \in W_{2}^{1 / 2}(\Gamma)$ существует единственное решение $f(z) \in W_{2}^{1}(\Gamma)$ задачи (6.7)-(6.9) или, что то же, исходной задачи минимизации коаналитического уклонения.

ДокАЗАТЕЛЬСТво. По своему построению задача (6.7)-(6.9) эквивалентна задаче разрешимости интегрального тождества

$$
\iint_{G}\left[\nabla\left(f-f_{a}\right) \nabla\left(\bar{\varphi}-\bar{\varphi}_{a}\right)+\left(f-f_{a}\right)\left(\bar{\varphi}-\bar{\varphi}_{a}\right)\right] d x d y=0, \quad \varphi(z) \in \stackrel{\circ}{W}_{2}^{1}(G),
$$

относительно функции $f(z) \in W_{2}^{1}(G)$, принимающей заданное значение на границе:

$$
\left.f(z)\right|_{\Gamma}=f_{0}(\gamma) \in W_{2}^{1 / 2}(\Gamma)
$$

Единственность решения устанавливается стандартно. Действительно, пусть $f(z)$ и $g(z)$ - два решения задачи (6.10)-(6.11). Тогда для разности $u(z)=f(z)-g(z)$ будем иметь тождество

$$
\iint_{G}\left[\nabla\left(u-u_{a}\right) \nabla\left(\bar{\varphi}-\bar{\varphi}_{a}\right)+\left(u-u_{a}\right)\left(\bar{\varphi}-\bar{\varphi}_{a}\right)\right] d x d y=0
$$


где $\varphi(z) \in \stackrel{\circ}{W} \frac{1}{2}(G)$ произвольна. Так как $\left.u(z)\right|_{\Gamma}=0$, то положив $\varphi(z)=u(z)$, немедленно получаем, что коаналитическая часть функции $u(z)$ равна нулю, т.е.

$$
f_{c a}(z)=g_{c a}(z), \quad z \in G .
$$

Поскольку функции $f(z)$ и $g(z)$ совпадают на границе Г, отсюда следует, что аналитические составляющие этих функций также равны на границе, т.е.

$$
\left.f_{a}(z)\right|_{\Gamma}=\left.g_{a}(z)\right|_{\Gamma}
$$

Ясно теперь, что $f_{a}(z) \equiv g_{a}(z)$ и в области $G$, а тем самым $f(z) \equiv g(z)$ для всех $z \in G$. Единственность установлена.

Сушествование решения установим методом Галеркина. Для этого прежде всего сведем задачу к случаю нулевого значения на границе. Именно, пусть $h(z) \in W_{2}^{1}(G)$ - какое-либо фиксированное продолжение функции $f_{0}(\gamma) \in W_{2}^{1 / 2}(\Gamma)$, т.е. $\left.h(z)\right|_{\Gamma}=$ $f_{0}(\gamma)$. Положим $u(z)=f(z)-h(z)$. Тогда неизвестная функция $u(z) \in \stackrel{\circ}{W} \frac{1}{2}(G)$ должна удовлетворять интегральному тождеству

$$
\iint_{G}\left[\nabla\left(u_{c a}+h_{c a}\right) \nabla \bar{\varphi}_{c a}(z)+\left(u_{c a}+h_{c a}\right) \bar{\varphi}_{c a}(z)\right] d x d y=0, \quad \varphi(z) \in \stackrel{\circ}{W}_{2}^{1}(G),
$$

где $u_{c a}(z), h_{c a}(z)$ и $\varphi_{c a}(z)$ - коаналитические части функций $u(z), h(z)$ и $\varphi(z)$.

Пусть $\varphi^{1}(z), \varphi^{2}(z), \ldots$ - базисная системав $\stackrel{\circ}{W} \underset{2}{1}(G)$. Приближенное решение $u^{N}(z)$, $N=1,2, \ldots$, ишем в виде

$$
u^{N}(z)=c_{N}^{1} \varphi^{1}(z)+\cdots+c_{N}^{N} \varphi^{N}(z),
$$

при этом неизвестные постоянные определяются из моментных соотношений Галеркина

$$
\iint_{G}\left[\nabla\left(u_{c a}^{N}+h_{c a}\right) \nabla \bar{\varphi}_{c a}^{j}+\left(u_{c a}^{N}+h_{c a}\right) \bar{\varphi}_{c a}^{j}\right] d x d y=0, \quad j=1,2, \ldots, N
$$

(обозначения ясны).

Система $\left(6.12^{N}\right)$ однозначно разрешима, при этом для коаналитических составляющих приближенных решений стандартно имеем оценку

$$
\left\|u_{c a}^{N}(z)\right\|_{W_{2}^{1}(G)} \leqslant M
$$

где $M=M(h)$ - постоянная, не зависящая от $N$.

Нетрудно видеть, что и для аналитических составляющих $u_{a}^{N}(z)$ справедлива аналогичная оценка. Действительно, из оценки (6.13) и известных неравенств теории следов функций из соболевских пространств вытекает, что для всех $N=1,2, \ldots$

$$
\left\|u_{c a}^{N}(\gamma)\right\|_{W_{2}^{1 / 2}(\Gamma)} \leqslant M\left\|u_{c a}^{N}(z)\right\|_{W_{2}^{1}(G)} \leqslant M_{1}
$$

где $M_{1}>0-$ постоянная. Отсюда, учитывая, что

$$
u^{N}(\gamma) \equiv u_{a}^{N}(\gamma)+u_{c a}^{N}(\gamma)=0, \quad \gamma \in \Gamma,
$$


получаем, что и

$$
\left\|u_{a}^{N}(\gamma)\right\|_{W_{2}^{1 / 2}(\Gamma)} \leqslant M_{2}
$$

где $M_{2}>0$ не зависит от $N$.

Поскольку всякая аналитическая функция является и гармонической (комплекснозначной), то, используя известные $L_{2}$-оценки теории эллиптических граничных задач, приходим к неравенству

$$
\left\|u_{a}^{N}(z)\right\|_{W_{2}^{1}(G)} \leqslant M_{3}\left\|u_{a}^{N}(\gamma)\right\|_{W_{2}^{1 / 2}(\Gamma)} \leqslant M_{4}
$$

В итоге получаем оценку

$$
\left\|u^{N}(z)\right\|_{W_{2}^{1}(G)} \leqslant M_{5}<\infty .
$$

Далее обычными рассуждениями устанавливается, что слабо предельная точка $u(z)$ последовательности $u^{N}(z)$ удовлетворяет интегральному тождеству (6.12). Тем самым решение $f(z)=h(z)+u(z)$ найдено.

Теорема доказана.

\section{4. Пример (еще об одном свойстве гармонической функции).}

Пусть $G=K$ - единичньй круг. Рассмотрим разобранную вьше задачу для круга в случае “чисто” градиентной метрики, т.е. для коаналитического уклонения

$$
\mu_{0}(f)=\iint_{K}\left|\nabla\left(f-f_{a}\right)\right|^{2} d x d y
$$

В этом случае задача о наименьшем коаналитическом уклонении имеет вид

$$
\begin{gathered}
\Delta f(z) \in \mathscr{O}_{2}^{-1}(G), \\
P_{+}\left\{\left.\frac{\partial}{\partial n}\left(f-f_{a}\right)\right|_{\Gamma}\right\}=0,\left.\quad f(z)\right|_{|z|=1}=f_{0}(\arg z),
\end{gathered}
$$

где $P_{+}-$проектор на подпространство $2 \pi$-периодических функций, ряд Фурье которых содержит экспоненты с неотрицательными частотами.

Можно показать (мы опускаем соответствующие рассуждения), что последняя задача эквивалентна задаче Дирихле и, следовательно, для круга продолжение с наименьшим уклонением $\mu_{0}(f)$ есть обычное гармоническое продолжение.

Автору неизвестно, верно ли это для любой односвязной области. В то же время прямой подсчет решения задачи о наименьшем коаналитическом продолжении в случае кольца показьвает, что оно не является гармоническим продолжением. 


\section{СПИСОК ЛИТЕРАТУРЫ}

[1] Математическая энциклопедия. М.: Советская энциклопедия, 1977.

[2] Трикоми Ф. Дифференциальные уравнения. М.: ИЛ., 1962.

[3] Маркушевич А.И. Теория аналитических функций. Т. 3. М.: Наука, 1968.

[4] Browder F. E. Nonlinear elliptic boundary value problems // Bull. Amer. Math. Soc. 1963. V. 69. №6. P. 862-874.

[5] Лионс ЖК. Некоторые методы решения нелинейных краевых задач. М.: Мир, 1972.

[6] Дубинский Ю. А. Нелинейные эллиптические и параболические уравнения любого порядка // УМН. 1968. Т. 23. №1. С. 45-90.

[7] Лаврентьев М.А., Шабат Б. В. Методы теории функций комплексного переменного. М.: Физматгиз, 1958.

[8] Джураев А. К теории эллиптических систем в многосвязных областях на плоскости // ДАН СССР. 1991. Т. 317. №6. С. 1294-1297.

[9] Хермандер Л. Введение в теорию функций многих комплексных переменных. М.: Мир, 1965.

[10] Харди Г., Литтлвуд Дж., Полиа Г. Неравенства. М.: ГИИЛ, 1948.

[11] Никольский С. М. Приближение функций многих переменных и теоремы вложения. М.: Наука, 1969.

[12] Сансоне Дж. Обыкновенные дифференциальные уравнения. М.: ИЛ, 1953.

[13] Дубинский Ю. А. Задача Коши и псевдодифференциальные операторы в комплексной области // УМН. 1990. Т. 45. № 2. С. 115-142.

[14] Dubinskii Ju. A. Analytic Pseudodifferential operators and their applications. Dordrecht-Boston-London: Klüwer Acad. Publ, 1991.

[15] Дубинский Ю. А. Об аналитической нелинейной периодической задаче // Дифф. уравнения. 1993. Т. 29. № 3. С. 371-380.

[16] Дубинский Ю.А.О периодической задаче для нелинейных эллиптических уравнений с комплексными аргументами // ДАН. 1993. Т. 330. № 2. С. 148-150.

[17] Дубинский Ю. А. Об одной нелинейной аналитической задаче // ДАН. 1994. Т. 332. № 5. С. 556-559.

[18] Дубинский Ю.А. Об аналитическом продолжении решений обькновенных дифференциальных уравнений // Дифф. уравнения. 1994. Т. 30. № 4. С. 725-728.

[19] Дубинский Ю.А. О корректности задачи Коши в комплексной области // Труды МИРАН. 1994. Т. 203. С. 78-90.

[20] Дубинский Ю. А. Об аналитической нелинейной периодической задаче для параболического уравнения // Вестник МЭИ. 1994.№ 4. С. 27-42.

Московский энергетический институт

Поступила в редакцию

(технический университет)

18.02.1996 